TRANSACTIONS OF THE

AMERICAN MATHEMATICAL SOCIETY

Volume 360, Number 6, June 2008, Pages 3263-3285

S 0002-9947(07)04432-7

Article electronically published on November 28, 2007

\title{
HARNACK-THOM THEOREM FOR HIGHER CYCLE GROUPS AND PICARD VARIETIES
}

\author{
JYH-HAUR TEH
}

\begin{abstract}
We generalize the Harnack-Thom theorem to relate the ranks of the Lawson homology groups with $\mathbb{Z}_{2}$-coefficients of a real quasiprojective variety with the ranks of its reduced real Lawson homology groups. In the case of zero-cycle group, we recover the classical Harnack-Thom theorem and generalize the classical version to include real quasiprojective varieties. We use Weil's construction of Picard varieties to construct reduced real Picard groups, and Milnor's construction of universal bundles to construct some weak models of classifying spaces of some cycle groups. These weak models are used to produce long exact sequences of homotopy groups which are the main tool in computing the homotopy groups of some cycle groups of divisors. We obtain some congruences involving the Picard number of a nonsingular real projective variety and the rank of its reduced real Lawson homology groups of divisors.
\end{abstract}

\section{INTRODUCTION}

In $[3,6,10]$, Friedlander and Lawson constructed Lawson homology and morphic cohomology, which serve as an enrichment of singular homology and singular cohomology, respectively, for complex projective varieties. In [18], the author constructed parallel theories for real projective varieties which are called reduced real Lawson homology and reduced real morphic cohomology. They enjoy many nice properties such as the Lawson suspension property, the homotopy invariance property, the bundle projection property, the splitting properties, and for each theory there exists a localization long exact sequence. By using the Friedlander-Lawson moving lemma (see [7]), it is shown that there is a duality theorem between Lawson homology and morphic cohomology (see [8]) and a duality theorem between reduced real Lawson homology and reduced real morphic cohomology (see [18]). Furthermore, this duality is compatible with Poincaré duality.

The Harnack theorem says that a nonsingular totally real curve of degree $d$ in $\mathbb{R P}^{2}$ has at most $g(d)+1$ connected components where $g(d)=\frac{(d-1)(d-2)}{2}$. Later on Thom generalized Harnack's result to a statement which says that for a real projective variety $X$, the total Betti number $B(X), B(R e X)$ and the Euler characteristic $\chi(X), \chi(\operatorname{Re} X)$ in $\mathbb{Z}_{2}$-coefficients of $X$ and the real points $\operatorname{Re} X$ of $X$ respectively satisfy the following relations (see $[2,9,19])$ :

$$
B(\operatorname{Re} X) \leq B(X), B(\operatorname{Re} X) \equiv B(X) \bmod 2, \chi(\operatorname{Re} X) \equiv \chi(X) \bmod 2 .
$$

Received by the editors May 9, 2006 and, in revised form, September 20, 2006.

2000 Mathematics Subject Classification. Primary 14C25, 14P25; Secondary 55Q52, 55N35.

Key words and phrases. Harnack-Thom theorem, algebraic cycles, Lawson homology, homotopy groups, Picard varieties, classifying spaces. 
In section 2 we give an overview of Lawson homology and reduced real Lawson homology. In section 3 we prove a splitting theorem which is the core of the proof of our main theorem. In section 4 we extend the classical Harnack-Thom theorem to a statement involving the ranks of Lawson homology groups with $\mathbb{Z}_{2}$-coefficients and the ranks of reduced real Lawson homology groups. For 0-cycle groups, we recover the Harnack-Thom theorem and generalize it to real quasiprojective varieties, in which case we need to use Borel-Moore homology instead of singular homology. To construct some nontrivial examples, we apply Weil's construction of Picard varieties to construct reduced real Picard groups in section 5. In section 6 we prove a vanishing theorem for the reduced real Lawson homology groups of divisors and under some mild conditions, and we get the following result by applying our main theorem from section 4 :

$$
\rho(X)+1 \equiv \operatorname{dim}_{\mathbb{Z}_{2}} \pi_{0} R_{m-1}(X)+\operatorname{dim}_{\mathbb{Z}_{2}} \pi_{1} R_{m-1}(X) \bmod 2
$$

where $\rho(X)$ is the Picard number of $X$.

The results of this paper suggest that Lawson homology and reduced real Lawson homology are useful enrichments of singular homology.

\section{Review of LAWson homology and REDUCEd REAL LAWSON HOMOLOGY}

Let us recall some basic properties of Lawson homology and reduced real Lawson homology (see $[3,10,18])$. For a projective variety $X$, denote the set of effective $p$-cycles of degree $d$ by $\mathscr{C}_{p, d}(X)$. By the Chow theorem (see $\left.[16]\right), \mathscr{C}_{p, d}(X)$ can be realized as a complex projective variety. With the analytic topology on $\mathscr{C}_{p, d}(X)$, we get a compact topological space $K_{p, d}(X)=\coprod_{d_{1}+d_{2} \leq d} \mathscr{C}_{p, d_{1}}(X) \times \mathscr{C}_{p, d_{2}}(X) / \sim$ where $\sim$ is the equivalence relation defined by $(a, b) \sim(c, d)$ if and only if $a+d=b+c$. These spaces form a filtration:

$$
K_{p, 0}(X) \subset K_{p, 1}(X) \subset K_{p, 2}(X) \subset \cdots=Z_{p}(X)
$$

where $Z_{p}(X)$ is the naive group completion of the monoid $\mathscr{C}_{p}(X)=\coprod_{d \geq 0} \mathscr{C}_{p, d}(X)$. We give $Z_{p}(X)$ the weak topology defined by this filtration, i.e., $U \subset Z_{p}(X)$ is open if and only if $U \cap K_{p, d}(X)$ is open for all $d$. We define the $n$-th Lawson homology group of $p$-cycles to be

$$
L_{p} H_{n}(X)=\pi_{n-2 p} Z_{p}(X),
$$

the $(n-2 p)$-th homotopy group of $Z_{p}(X)$. We define the $n$-th Lawson homology group with $\mathbb{Z}_{2}$-coefficients of $p$-cycles to be

$$
L_{p} H_{n}\left(X ; \mathbb{Z}_{2}\right)=\pi_{n-2 p}\left(\frac{Z_{p}(X)}{2 Z_{p}(X)}\right) .
$$

For the zero-cycle group, by the Dold-Thom theorem, we have an isomorphism

$$
L_{0} H_{n}\left(X ; \mathbb{Z}_{2}\right)=H_{n}\left(X ; \mathbb{Z}_{2}\right)
$$

between Lawson homology and singular homology.

For a quasiprojective variety $U$, there exist projective varieties $X$ and $Y$ where $Y \subset X$ such that $U=X-Y$. The Lawson homology group of $U$ is defined to be

$$
L_{p} H_{n}(U)=\pi_{n-2 p}\left(\frac{Z_{p}(X)}{Z_{p}(Y)}\right) \text {. }
$$


It is proved in [12] that this definition is independent of the choice of $X$ and $Y$. We define Lawson homology with $\mathbb{Z}_{2}$-coefficients of $U$ to be

$$
L_{p} H_{n}\left(U ; \mathbb{Z}_{2}\right)=\pi_{n-2 p}\left(\frac{\frac{Z_{p}(X)}{2 Z_{p}(X)}}{\frac{Z_{p}(Y)}{2 Z_{p}(Y)}}\right)=\pi_{n-2 p}\left(\frac{\frac{Z_{p}(X)}{Z_{p}(Y)}}{2 \frac{Z_{p}(X)}{Z_{p}(Y)}}\right)=\pi_{n-2 p}\left(\frac{\frac{Z_{p}(X)}{Z_{p}(Y)}}{\frac{2 Z_{p}(X)}{2 Z_{p}(Y)}}\right) .
$$

For the zero-cycle group, by the Dold-Thom theorem (see [4] Proposition 1.6), we have an isomorphism

$$
L_{0} H_{n}(U)=H_{n}^{B M}(U) \text { and } L_{0} H_{n}\left(U ; \mathbb{Z}_{2}\right)=H_{n}^{B M}\left(U ; \mathbb{Z}_{2}\right)
$$

where $H_{*}^{B M}$ denotes Borel-Moore homology.

A real projective variety $X \subset \mathbb{P}^{n}$ is a complex projective variety which is invariant under conjugation. Equivalently, it is a complex projective variety defined by some real polynomials. Conjugation induces a $\mathbb{Z}_{2}$-action on $Z_{p}(X)$. Let $Z_{p}(X)_{\mathbb{R}}$ be the subgroup of $p$-cycles on $X$ which are invariant under this action and let $Z_{p}(X)^{a v}$ be the subgroup consisting of cycles of the form $c+\bar{c}$ where $c \in Z_{p}(X)$ and $\bar{c}$ is the conjugate cycle of $c$. These two subgroups are endowed with the subspace topology. Define the reduced real $p$-cycle group to be

$$
R_{p}(X)=\frac{Z_{p}(X)_{\mathbb{R}}}{Z_{p}(X)^{a v}}
$$

It is shown in [18], Proposition 2.4 that $Z_{p}(X)^{a v}$ is a closed subgroup of $Z_{p}(X)_{\mathbb{R}}$ and in the Appendix of [18] that all these cycle groups $Z_{p}(X), Z_{p}(X)_{\mathbb{R}}, Z_{p}(X)^{a v}, R_{p}(X)$ are $\mathrm{CW}$-complexes. We define the $n$-th reduced real Lawson homology group of $p$-cycles to be

$$
R L_{p} H_{n}(X)=\pi_{n-p} R_{p}(X) .
$$

For zero cycles, it is shown in [18], Proposition 2.8 that $R L_{0} H_{n}(X)=H_{n}\left(\operatorname{Re} X ; \mathbb{Z}_{2}\right)$, the singular homology group of the real points of $X$.

We define the reduced real Lawson homology group of a real quasiprojective variety $U=X-Y$ to be

$$
R L_{p} H_{n}(U)=\pi_{n-p}\left(\frac{R_{p}(X)}{R_{p}(Y)}\right)
$$

where $X, Y$ are real projective varieties and $Y \subset X$. It is shown in [18] that this definition is independent of the choice of $X, Y$. For the group of zero cycles, we have $R L_{o} H_{n}(U)=\pi_{n}\left(\frac{R_{0}(X)}{R_{0}(Y)}\right)=H_{n}^{B M}\left(R e U ; \mathbb{Z}_{2}\right)$.

\section{THE SPLITTING THEOREM}

Let us recall that the real part $R P(C)$ of a cycle $C$, roughly speaking, is the part consisting of irreducible real subvarieties, and the averaged part $A P(C)$ of a cycle $C$ is the part consisting of conjugate pairs of complex cycles. The imaginary part is the part left after canceling out the real and averaged parts. We give the precise definition in the following:

Definition 3.1. For any $f \in Z_{p}(X)$, let $f=\sum_{i \in I} n_{i} V_{i}$ be in the reduced form, i.e., each $V_{i}$ is an irreducible subvariety of $X$ and $V_{i}=V_{j}$ if and only if $i=j$. Let

$$
R P(f)=\sum_{i \in I, \overline{V_{i}}=V_{i}} n_{i} V_{i}
$$


which is called the real part of $f$. Let

$$
J=\left\{i \in I \mid V_{i} \text { is not real and } \overline{V_{i}} \text { is also a component of } f\right\}
$$

and for $i \in J$, let $m_{i}$ be the maximum value of the coefficients of $V_{i}$ and $\overline{V_{i}}$. Define the averaged part to be

$$
A P(f)=\sum_{i \in J} m_{i}\left(V_{i}+\bar{V}_{i}\right)
$$

and the imaginary part to be

$$
I P(f)=f-R P(f)-A P(f) .
$$

It is easy to see that $f$ is a real cycle if and only if $I P(f)=0$ and a real cycle $g$ is an averaged cycle if and only if $R P(g)$ is divisible by 2 .

In the following, we will assume that $X$ is a real projective variety.

Proposition 3.2. The following sequence is exact:

$$
0 \longrightarrow \frac{Z_{p}(X)_{\mathbb{R}}}{2 Z_{p}(X)_{\mathbb{R}}} \stackrel{i}{\longrightarrow} \frac{Z_{p}(X)}{2 Z_{p}(X)} \stackrel{1+c_{*}}{\longrightarrow} \frac{Z_{p}(X)^{a v}}{2 Z_{p}(X)_{\mathbb{R}}} \longrightarrow 0 .
$$

Proof. It is easy to check that $i\left(f+2 Z_{p}(X)_{\mathbb{R}}\right)=f+2 Z_{p}(X)$ is well defined and injective and $\left(1+c_{*}\right)\left(f+2 Z_{p}(X)\right)=f+\bar{f}+2 Z_{p}(X)_{\mathbb{R}}$ is well defined and surjective. The map $1+c_{*}$ sends the image of $i$ to 0 , thus the only thing we need to prove is for $f+\bar{f} \in 2 Z_{p}(X)_{\mathbb{R}}, f$ is in $Z_{p}(X)_{\mathbb{R}}$. Since $f+\bar{f}=2 R P(f)+2 A P(f)+I P(f)+I P(\bar{f}) \in$ $2 Z_{p}(X)_{\mathbb{R}}$, this implies that $I P(f)=I P(\bar{f})=0$, so $f \in Z_{p}(X)_{\mathbb{R}}$.

Definition 3.3. Let $Q_{p}(X)$ be the collection of all averaged cycles $c$ such that there exists a sequence $\left\{v_{i}\right\} \subset Z_{p}(X)_{\mathbb{R}}$ where $v_{i}=R P\left(v_{i}\right)$ for all $i$ and $v_{i}$ converges to $c$. It is not difficult to see that $Q_{p}(X)$ is a topological subgroup of $Z_{p}(X)^{a v}$. Let $Z Q_{p}(X)_{\mathbb{R}}=2 Z_{p}(X)_{\mathbb{R}}+Q_{p}(X)$ denote the internal sum of $2 Z_{p}(X)_{\mathbb{R}}$ and $Q_{p}(X)$. Then $Z Q_{p}(X)_{\mathbb{R}}$ is again a topological subgroup of $Z_{p}(X)^{a v}$. The group $Q_{p}(X)$ is the intersection of the closure of the group formed by irreducible real $p$-subvarieties with the averaged $p$-cycle group. Thus $Z Q_{p}(X)_{\mathbb{R}}$ is a closed subgroup.

Proposition 3.4. For a real projective variety $X, Z Q_{0}(X)_{\mathbb{R}}=2 Z_{0}(X)_{\mathbb{R}}$.

Proof. The free abelian group $Z_{0}(\operatorname{Re} X)$ generated by real points of $X$ is closed in $Z_{0}(X)$, so if $c \in Q_{0}(X)$, then $c \in 2 Z_{0}(X)_{\mathbb{R}}$ (see Proposition 2.8 in [18]).

The following example was given by Lawson to show that the set of 1-cycles formed by irreducible real subvarieties may not be closed, which contrasts the case of 0 -cycles, i.e., $Z Q_{p}(X)_{\mathbb{R}}$ may not equal to $2 Z_{p}(X)_{\mathbb{R}}$ if $p>0$.

Example 3.5. In $\mathbb{P}^{2}$, consider the sequence of irreducible real subvarieties $V_{\epsilon}=$ zero locus of $X^{2}+Y^{2}-\epsilon Z^{2}$. As $\epsilon$ converges to $0, V_{\epsilon}$ converges to the cycle formed by two lines $X=i Y$ and $X=-i Y$ which is an averaged cycle but not in $2 Z_{p}(X)_{\mathbb{R}}$.

Lemma 3.6. Suppose that the sequence $\left\{A P\left(f_{i}\right)\right\}$ converges to $f$ where $f_{i} \in Z_{p}(X)$; then $R P(f) \in 2 Z_{p}(X)_{\mathbb{R}}$. 
Proof. Since $A P\left(f_{i}\right) \in Z_{p}(X)^{a v}$ and $Z_{p}(X)^{a v}$ is closed, $f$ is in $Z_{p}(X)^{a v}$ so $R P(f) \in$ $2 Z_{p}(X)_{\mathbb{R}}$.

Lemma 3.7. Define $\widetilde{A P}: \frac{Z_{p}(X)_{\mathbb{R}}}{Z Q_{p}(X)_{\mathbb{R}}} \longrightarrow \frac{Z_{p}(X)^{a v}}{Z Q_{p}(X)_{\mathbb{R}}} b y$

$$
f+Z Q_{p}(X)_{\mathbb{R}} \longmapsto A P(f)+Z Q_{p}(X)_{\mathbb{R}}
$$

Then $\widetilde{A P}$ is continuous.

Proof. There is a filtration called the canonical real filtration

$$
K_{1} \subset K_{2} \subset K_{3} \subset \cdots=Z_{p}(X)_{\mathbb{R}}
$$

where each $K_{i}$ is compact and the topology of $Z_{p}(X)_{\mathbb{R}}$ is given by the weak topology induced from this filtration. Thus the filtration

$$
K_{1}+Z Q_{p}(X)_{\mathbb{R}} \subset K_{2}+Z Q_{p}(X)_{\mathbb{R}} \subset \cdots=\frac{Z_{p}(X)_{\mathbb{R}}}{Z Q_{p}(X)_{\mathbb{R}}}
$$

defines the topology of $\frac{Z_{p}(X)_{\mathbb{R}}}{Z Q_{p}(X)_{\mathbb{R}}}$.

To show that $\widetilde{A P}$ is continuous, it suffices to show that $\widetilde{A P}$ maps convergent sequences to convergent sequences. The reason is as follows: by elementary pointset topology, $\widetilde{A P}$ is continuous if and only if $\widetilde{A P}(\bar{A}) \subset \overline{\widehat{A P}(A)}$ for any subset $A \subset \frac{Z_{p}(X)_{\mathbb{R}}}{Z Q_{p}(X)_{\mathbb{R}}}$ where $\bar{A}$ is the closure of $A$. Let $\pi: Z_{p}(X)_{\mathbb{R}} \rightarrow \frac{Z_{p}(X)_{\mathbb{R}}}{Z Q_{p}(X)_{\mathbb{R}}}$ be the quotient map. For any subset $A \subset \frac{Z_{p}(X)_{\mathbb{R}}}{Z Q_{p}(X)_{\mathbb{R}}}$, since $\pi$ is an open map, we have $\pi^{-1}(\bar{A}) \subset \overline{\pi^{-1}(A)}$ where $\bar{A}$ is the closure of $A$. Hence for $x \in \bar{A}$, there is $y \in \overline{\pi^{-1}(A)}$ such that $\pi(y)=x$. Since $Z_{p}(X)_{\mathbb{R}}$ is a $\mathrm{CW}$-complex (actually it is also a metric space), there is $y_{n} \in \pi^{-1}(A)$ such that $y_{n} \rightarrow y$. If $\widetilde{A P}$ maps convergent sequences to convergent sequences, we see that $\widetilde{A P}\left(\pi\left(y_{n}\right)\right)$ converges to $\widetilde{A P}(x)$. Since $\pi\left(y_{n}\right) \in A$ and $\pi(y)=x$, we have $\widetilde{A P}(x) \in \overline{\widetilde{A P}(A)}$ which implies that $\widetilde{A P}$ is continuous.

Suppose that $f_{i}+Z Q_{p}(X)_{\mathbb{R}}$ converges to $Z Q_{p}(X)_{\mathbb{R}}$. Since $A=\left\{f_{i}+Z Q_{p}(X)_{\mathbb{R}}\right\} \cup$ $\left\{Z Q_{p}(X)_{\mathbb{R}}\right\}$ is compact and $\frac{Z_{p}(X)_{\mathbb{R}}}{Z Q_{p}(X)_{\mathbb{R}}}$ is Hausdorff, by Lemma 2.3 in [18], $A \subset$ $K_{n}+Z Q_{p}(X)_{\mathbb{R}}$ for some $n$. Thus there exists $g_{i} \in K_{n}$ such that under the quotient map $q, q\left(g_{i}\right)=f_{i}+Z Q_{p}(X)_{\mathbb{R}}$ for all $i$. The set $K_{n}$ is compact, thus $\left\{g_{i}\right\}$ has a convergent subsequence.

Let $\left\{g_{i j}\right\}$ be a subsequence of $\left\{g_{i}\right\}$ which converges to $g$. Since $g_{i j}+Z Q_{p}(X)_{\mathbb{R}}$ converges to $Z Q_{p}(X)_{\mathbb{R}}$, we have $g \in Z Q_{p}(X)_{\mathbb{R}}$. The set $\left\{g_{i j}\right\} \subset K_{n}$ and each $g_{i j}$ is a real cycle which implies that $\left\{A P\left(g_{i j}\right)\right\} \subset K_{n}$ and hence $\left\{A P\left(g_{i j}\right)\right\}$ has a convergent subsequence. Let $\left\{A P\left(g_{i j k}\right)\right\}$ be a subsequence of $\left\{A P\left(g_{i j}\right)\right\}$ which converges to a real cycle $h$. Since $\left\{g_{i j k}\right\}$ is a subsequence of $\left\{g_{i j}\right\}$, it converges to $g$, hence

$$
R P\left(g_{i j k}\right)=g_{i j k}-A P\left(g_{i j k}\right) \longrightarrow g-h .
$$

By Lemma 3.6, $R P(h) \in 2 Z_{p}(X)_{\mathbb{R}}$, and since $R P(g) \in 2 Z_{p}(X)_{\mathbb{R}}$ we have $R P(g-h) \in 2 Z_{p}(X)_{\mathbb{R}}$. The cycle $g-h$ is a real cycle and this implies $g-h \in$ $Z_{p}(X)^{a v}$. Furthermore, since $\left\{R P\left(g_{i j k}\right)\right\} \longrightarrow g-h$, by definition, $g-h \in Q_{p}(X) \subset$ $Z Q_{p}(X)_{\mathbb{R}}$. The cycle $g$ is in $Z Q_{p}(X)_{\mathbb{R}}$, thus $h \in Z Q_{p}(X)_{\mathbb{R}}$. Passing to the quotient, we see that $A P\left(g_{i j k}\right)+Z Q_{p}(X)_{\mathbb{R}} \longrightarrow h+Z Q_{p}(X)_{\mathbb{R}}=Z Q_{p}(X)_{\mathbb{R}}$ for any convergent 
subsequence $\left\{A P\left(g_{i j k}\right)\right\}$ of $\left\{A P\left(g_{i j}\right)\right\}$, thus $A P\left(g_{i j}\right)+Z Q_{p}(X)_{\mathbb{R}} \longrightarrow Z Q_{p}(X)_{\mathbb{R}}$. For any convergent subsequence $\left\{g_{i j}\right\}$ of $\left\{g_{i}\right\}, \widetilde{A P}\left(g_{i j}+Z Q_{p}(X)_{\mathbb{R}}\right)=A P\left(g_{i j}\right)+$ $Z Q_{p}(X)_{\mathbb{R}}$ converges to the point $Z Q_{p}(X)_{\mathbb{R}}$. Consequently, this implies that

$$
\widetilde{A P}\left(g_{i}+Z Q_{p}(X)_{\mathbb{R}}\right)=\widetilde{A P}\left(f_{i}+Z Q_{p}(X)_{\mathbb{R}}\right)
$$

converges to $Z Q_{p}(X)_{\mathbb{R}}$. So $\widetilde{A P}$ is continuous.

Lemma 3.8. Define $\widetilde{R P}: \frac{Z_{p}(X)_{\mathbb{R}}}{Z_{p}(X)^{a v}} \longrightarrow \frac{Z_{p}(X)_{\mathbb{R}}}{Z Q_{p}(X)_{\mathbb{R}}} b y$

$$
f+Z_{p}(X)^{a v} \longmapsto R P(f)+Z Q_{p}(X)_{\mathbb{R}}
$$

Then $\widetilde{R P}$ is continuous.

Proof. We proceed as in the proof above. The canonical real filtration

$$
K_{1} \subset K_{2} \subset K_{3} \subset \cdots=Z_{p}(X)_{\mathbb{R}}
$$

induces a filtration

$$
K_{1}+Z_{p}(X)^{a v} \subset K_{2}+Z_{p}(X)^{a v} \subset \cdots=\frac{Z_{p}(X)_{\mathbb{R}}}{Z_{p}(X)^{a v}}
$$

which defines the topology of $\frac{Z_{p}(X)_{\mathbb{R}}}{Z_{p}(X)^{a v}}$ and the filtration

$$
K_{1}+Z Q_{p}(X)_{\mathbb{R}} \subset K_{2}+Z Q_{p}(X)_{\mathbb{R}} \subset \cdots=\frac{Z_{p}(X)_{\mathbb{R}}}{Z Q_{p}(X)_{\mathbb{R}}}
$$

which defines the topology of $\frac{Z_{p}(X)_{\mathbb{R}}}{Z Q_{p}(X)_{\mathbb{R}}}$.

By an argument similar to that of the previous proof, it suffices to prove that $\widetilde{R P}$ maps convergent sequences to convergent sequences. Suppose that $f_{i}+Z_{p}(X)^{a v}$ converges to $Z_{p}(X)^{a v}$. Since $A=\left\{f_{i}+Z_{p}(X)^{a v}\right\} \cup\left\{Z_{p}(X)^{a v}\right\}$ is compact, we have $A \subset K_{n}+Z_{p}(X)^{a v}$ for some $n$. Thus there exists $g_{i} \in K_{n}$ such that under the quotient map $q, q\left(g_{i}\right)=f_{i}+Z_{p}(X)^{a v}$ for all $i$. Let $\left\{g_{i j}\right\}$ be a subsequence of $\left\{g_{i}\right\}$ which converges to $g$. Since $g_{i j}+Z_{p}(X)^{a v}$ converges to $Z_{p}(X)^{a v}$, this implies that $g \in Z_{p}(X)^{a v}$. The set $\left\{g_{i j}\right\} \subset K_{n}$ implies that $\left\{R P\left(g_{i j}\right)\right\} \subset K_{n}$, thus $\left\{R P\left(g_{i j}\right)\right\}$ has a convergent subsequence. Let $\left\{R P\left(g_{i j k}\right)\right\}$ be a subsequence of $\left\{R P\left(g_{i j}\right)\right\}$ which converges to a real cycle $h$. Since $\left\{g_{i j k}\right\}$ is a subsequence of $\left\{g_{i j}\right\}$, it converges to $g$, hence

$$
A P\left(g_{i j k}\right)=g_{i j k}-R P\left(g_{i j k}\right) \longrightarrow g-h .
$$

By Lemma 3.6, $R P(g-h) \in 2 Z_{p}(X)_{\mathbb{R}}$, and since $R P(g) \in 2 Z_{p}(X)_{\mathbb{R}}$ we have $R P(h) \in 2 Z_{p}(X)_{\mathbb{R}}$. The cycle $h$ is a real cycle and thus $h \in Z_{p}(X)^{a v}$. Furthermore, $\left\{R P\left(g_{i j k}\right)\right\} \longrightarrow h$, so by definition, $h \in Q_{p}(X)$. Passing to the quotient, we see that $R P\left(g_{i j k}\right)+Z Q_{p}(X)_{\mathbb{R}} \longrightarrow h+Z Q_{p}(X)_{\mathbb{R}}=Z Q_{p}(X)_{\mathbb{R}}$. Thus 
$R P\left(g_{i j}\right)+Z Q_{p}(X)_{\mathbb{R}} \longrightarrow Z Q_{p}(X)_{\mathbb{R}}$. For every convergent subsequence $\left\{g_{i j}\right\}$ of $\left\{g_{i}\right\}$, $\widetilde{R P}\left(g_{i j}+Z_{p}(X)^{a v}\right)$ converges to the point $Z Q_{p}(X)_{\mathbb{R}}$, thus $\widetilde{R P}\left(g_{i}+Z_{p}(X)^{a v}\right) \longrightarrow$ $Z Q_{p}(X)_{\mathbb{R}}$ and therefore $\widetilde{R P}$ is continuous.

Theorem 3.9 (The splitting theorem). $\frac{Z_{p}(X)_{\mathbb{R}}}{Z Q_{p}(X)_{\mathbb{R}}}$ is isomorphic as a topological group to $\frac{Z_{p}(X)_{\mathbb{R}}}{Z_{p}(X)^{a v}} \times \frac{Z_{p}(X)^{a v}}{Z Q_{p}(X)_{\mathbb{R}}}$.

Proof. Define $\psi: \frac{Z_{p}(X)_{\mathbb{R}}}{Z Q_{p}(X)_{\mathbb{R}}} \longrightarrow \frac{Z_{p}(X)_{\mathbb{R}}}{Z_{p}(X)^{a v}} \times \frac{Z_{p}(X)^{a v}}{Z Q_{p}(X)_{\mathbb{R}}}$ by $f+Z Q_{p}(X)_{\mathbb{R}} \longmapsto$ $\left(f+Z_{p}(X)^{a v}, A P(f)+Z Q_{p}(X)_{\mathbb{R}}\right)$ and define $\phi: \frac{Z_{p}(X)_{\mathbb{R}}}{Z_{p}(X)^{a v}} \times \frac{Z_{p}(X)^{a v}}{Z Q_{p}(X)_{\mathbb{R}}} \longrightarrow \frac{Z_{p}(X)_{\mathbb{R}}}{Z Q_{p}(X)_{\mathbb{R}}}$ by $\left(f+Z_{p}(X)^{a v}, g+Z Q_{p}(X)_{\mathbb{R}}\right) \longmapsto R P(f)+g+Z Q_{p}(X)_{\mathbb{R}}$. By the two lemmas above, $\psi$ and $\phi$ are continuous and it is easy to check they are inverse to each other.

\section{The Generalized HarnacK-Thom theorem}

While it is easy to produce an exact sequence $H \hookrightarrow G \longrightarrow G / H$ of topological groups, it is cumbersome to verify that it is a locally trivial principal $H$-bundle, and worse, it may not be in general. But the long exact homotopy sequence induced by a fibration is extremely useful in homotopy group calculation. We use Milnor's construction of universal bundles to construct some weak models of the classifying spaces of some cycle groups. They are used to produce long exact sequences of homotopy groups. To make everything work out, we need to work in the category of compactly generated topological spaces $\mathcal{C G}$ (see [17]).

We recall that a space $X$ is compactly generated if and only if $X$ is Hausdorff and each subset $A$ of $X$ with the property that $A \cap C$ is closed for every compact subset $C$ of $X$ is itself closed. Since the topology of our cycle groups is defined by a filtration of compact Hausdorff spaces, all groups we are dealing with are in $\mathcal{C G}$. To make sure the quotient $G / H$ is in this category, we need $H$ to be a normal closed subgroup of $G$.

Let us recall Milnor's construction of universal $G$-bundles. We adopt the notation from page 36 of [5]. For a topological group $G$, let $C_{G}=(G \times I) /(G \times\{0\})$ be the cone on $G$, and the $n$-th join, $G^{* n}$, is the subspace of $C_{G} \times \cdots \times C_{G}$ of points $\left(\left(g_{0}, t_{0}\right), \ldots,\left(g_{n}, t_{n}\right)\right)$ such that $\sum t_{i}=1$. Thus $G^{* n} \subset G^{*(n+1)}$. For a topological group $G \in \mathcal{C} \mathcal{G}$, as in [5], we give $E(G)=\bigcup_{n} G^{* n}$ the weak topology determined by $G^{* n}$ instead of Milnor's strong topology for arbitrary topological groups. Then we have a continuous action of $G$ in $E(G)$ given by

$$
\left(\left(g_{0}, t_{0}\right), \ldots,\left(g_{n}, t_{n}\right)\right) \cdot g=\left(\left(g_{0} g, t_{0}\right), \ldots,\left(g_{n} g, t_{n}\right)\right) .
$$

Set $B(G)=E(G) / G$ and let $p_{G}: E(G) \rightarrow B(G)$ be the quotient map. Then

- $p_{G}: E(G) \rightarrow B(G)$ is a principal $G$-bundle.

- $\pi_{k}(E(G))=0$ for $k \geq 0$.

The space $B(G)$ is called the classifying space of $G$, and we have $\pi_{k+1}(B(G)) \cong$ $\pi_{k}(G)$. We say that a space $T$ is a weak model of $B G$ if $T$ is weak homotopy equivalent to $B G$, i.e, they have the same homotopy groups.

The following result is the main tool that we use to produce long exact sequences of homotopy groups. A similar argument for topological groups which are CWcomplexes can be found in Theorem 2.4.12 of [1]. 
Proposition 4.1. Let $H, G \in \mathcal{C G}$ be two topological abelian groups and $H$ be a closed subgroup of $G$. Then we have a long exact sequence of homotopy groups:

$$
\cdots \rightarrow \pi_{k+1}(G / H) \rightarrow \pi_{k}(H) \rightarrow \pi_{k}(G) \rightarrow \pi_{k}(G / H) \rightarrow \cdots .
$$

From this result, when we have a short exact sequence $0 \rightarrow H \rightarrow G \rightarrow K \rightarrow 0$ of topological abelian groups such that $K$ is isomorphic to $G / H$, it induces a long exact sequence of homotopy groups. By abuse of terminology, we will call this long exact sequence the homotopy sequence induced by the short exact sequence.

Proof. From the principal $H$-bundle

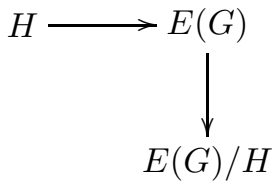

We see that $E(G) / H$ is a weak model of $B(H)$.

The group $G / H$, which is also in $\mathcal{C G}$ since $H$ is a closed subgroup, acts on $E(G) / H$, and we have $(E(G / H) \times E(G)) / G=(E(G / H) \times(E(G) / H)) /(G / H)$ which is a weak model of $B(G)$. We have a fibration

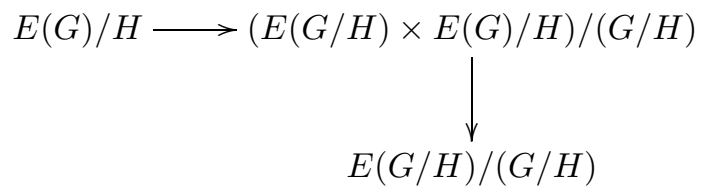

given by the first projection which induces a long exact sequence of homotopy groups

$$
\cdots \rightarrow \pi_{k+1}(G / H) \rightarrow \pi_{k}(H) \rightarrow \pi_{k}(G) \rightarrow \pi_{k}(G / H) \rightarrow \cdots .
$$

Note that since $H, G$ may not be $\mathrm{CW}$-complexes, the homomorphisms in the long exact sequence may not be induced by maps between $H$ and $G$.

We recall that for a surjective morphism $\phi: G \longrightarrow G^{\prime}$ between topological groups $G, G^{\prime}$, if $\phi$ is an open map or closed map, it induces an isomorphism of topological groups $G / \operatorname{Ker} \phi \cong G^{\prime}$. Also, for two normal subgroups $H_{1} \subset H_{2} \subset G$, we have $\left(G / H_{1}\right) /\left(H_{2} / H_{1}\right) \cong\left(G / H_{2}\right)$.

Let $A=\frac{Z_{p}(X)_{\mathbb{R}}}{2 Z_{p}(X)_{\mathbb{R}}}, B=\frac{Z_{p}(X)}{2 Z_{p}(X)}, C=\frac{Z_{p}(X)^{a v}}{2 Z_{p}(X)_{\mathbb{R}}}, D=\frac{Z_{p}(X)_{\mathbb{R}}}{Z Q_{p}(X)_{\mathbb{R}}}, E=\frac{Z_{p}(X)_{\mathbb{R}}}{Z_{p}(X)^{a v}}$, $F=\frac{Z_{p}(X)^{a v}}{Z Q_{p}(X)_{\mathbb{R}}}, G=\frac{Z Q_{p}(X)_{\mathbb{R}}}{2 Z_{p}(X)_{\mathbb{R}}}$.

Proposition 4.2. (1) The map $1+c_{*}: Z_{p}(X) \longrightarrow Z_{p}(X)^{\text {av }}$ is a closed map.

(2) The map $1+c_{*}: B \longrightarrow C$ is a closed map.

(3) The natural map $\phi: A \longrightarrow E$ is open.

(4) The natural map $\phi: A \longrightarrow D$ is open.

(5) The natural map $\phi: C \longrightarrow F$ is open.

Proof. (1) Let $K_{1} \subset K_{2} \subset \cdots \subset Z_{p}(X)$ be the canonical filtration and $K_{1}^{a v} \subset$ $K_{2}^{a v} \subset \cdots \subset Z_{p}(X)^{a v}$ be the canonical averaged filtration. Let $C \subset Z_{p}(X)$ be a closed set. Then $\left(1+c_{*}\right)(C) \cap K_{i}^{a v}=\left(1+c_{*}\right)\left(C \cap K_{\left[\frac{i}{2}\right]}\right)$ which is compact thus closed for all $i$. Therefore, $\left(1+c_{*}\right)(C)$ is closed. 
(2) Consider the following commutative diagram:

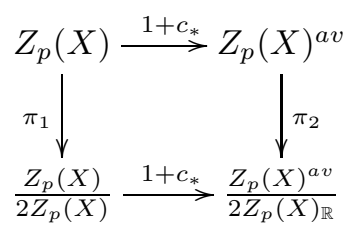

Let $C \subset \frac{Z_{p}(X)}{2 Z_{p}(X)}$ be a closed subset. Then $\pi_{1}^{-1}(C)$ is closed, which implies $\pi_{2}^{-1}\left(1+c_{*}\right)(C)=\left(1+c_{*}\right)\left(\pi_{1}^{-1}(C)\right)$ is closed. Since $\pi_{2}$ is a quotient map, it follows that $\left(1+c_{*}\right)(C)$ is closed.

(3) We have a commutative diagram

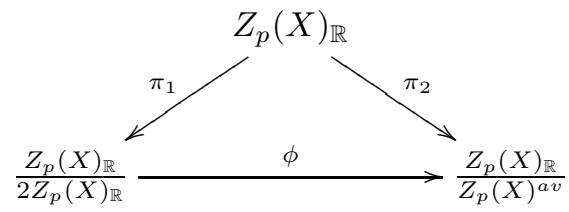

Since $\pi_{1}, \pi_{2}$ are open maps, $\phi$ is also an open map.

The proof of (4) and (5) are similar to the proof of (3).

In the following proposition we use the notation $T_{n}$ to denote the $n$-th homotopy group of $T$ where $T$ is any of the groups $A, \ldots, G$. We note that all these groups are $\mathbb{Z}_{2}$-spaces so their homotopy groups are vector spaces over $\mathbb{Z}_{2}$.

Proposition 4.3. We have the following short exact sequences:

(1) $0 \rightarrow A \longrightarrow B \longrightarrow C \rightarrow 0$,

(2) $0 \rightarrow C \longrightarrow A \longrightarrow E \rightarrow 0$,

(3) $0 \rightarrow G \longrightarrow A \longrightarrow D \rightarrow 0$,

(4) $0 \rightarrow G \longrightarrow C \longrightarrow F \rightarrow 0$.

They induce long exact sequences:

(1) $\cdots \stackrel{c_{n+1}}{\longrightarrow} A_{n} \stackrel{a_{n}}{\longrightarrow} B_{n} \stackrel{b_{n}}{\longrightarrow} C_{n} \stackrel{c_{n}}{\longrightarrow} A_{n-1} \longrightarrow \cdots$,

(2) $\cdots \stackrel{e_{n+1}}{\longrightarrow} C_{n} \stackrel{c_{n}^{\prime}}{\longrightarrow} A_{n} \stackrel{a_{n}^{\prime}}{\longrightarrow} E_{n} \stackrel{e_{n}}{\longrightarrow} C_{n-1} \longrightarrow \cdots$,

(3) $\cdots \stackrel{d_{n+1}}{\longrightarrow} G_{n} \stackrel{g_{n}}{\longrightarrow} A_{n} \stackrel{a_{n}^{\prime \prime}}{\longrightarrow} D_{n} \stackrel{d_{n}}{\longrightarrow} G_{n-1} \longrightarrow \cdots$,

(4) $\cdots \stackrel{f_{n+1}}{\longrightarrow} G_{n} \stackrel{g_{n}^{\prime}}{\longrightarrow} C_{n} \stackrel{c_{n}^{\prime \prime}}{\longrightarrow} F_{n} \stackrel{f_{n}}{\longrightarrow} G_{n-1} \longrightarrow \cdots$.

Proof. (1) By Proposition 3.2, $A$ is isomorphic to $k e r\left(1+c_{*}\right)$. The map $1+c_{*}$ is surjective and closed by Proposition 4.2, thus $C$ is isomorphic as a topological group to $B / \operatorname{ker}\left(1+c_{*}\right)$. Hence we have the first exact sequence, and by Proposition 4.1, we have the first homotopy sequence. A similar argument works for (2), (3) and (4).

Since every topological abelian group is a product of Eilenberg-MacLane spaces, we are able to compute the homotopy types of topological abelian groups from knowledge of their homotopy groups alone.

Example 4.4. The homotopy types of the seven groups mentioned above for 1cycles on $\mathbb{P}^{2}$ are $A=K\left(\mathbb{Z}_{2}, 0\right) \times K\left(\mathbb{Z}_{2}, 1\right) \times K\left(\mathbb{Z}_{2}, 2\right), B=K\left(\mathbb{Z}_{2}, 0\right) \times K\left(\mathbb{Z}_{2}, 2\right), C=$ $K\left(\mathbb{Z}_{2}, 2\right), D=E=K\left(\mathbb{Z}_{2}, 0\right) \times K\left(\mathbb{Z}_{2}, 1\right), F=0, G=C$. 
Definition 4.5. Suppose that $X$ is a real quasiprojective variety. We define the $L_{p}$-total Betti number of $X$ with $\mathbb{Z}_{2}$-coefficients to be

$$
B(p)(X)=\sum_{k=2 p}^{\infty} \operatorname{dim}_{\mathbb{Z}_{2}} L_{p} H_{k}\left(X ; \mathbb{Z}_{2}\right)
$$

where

$$
L_{p} H_{k}\left(X ; \mathbb{Z}_{2}\right)=\pi_{k-2 p}\left(\frac{Z_{p}(X)}{2 Z_{p}(X)}\right) .
$$

We define the real $L_{p}$-total Betti number to be $\beta(p)(X)=\sum_{k=p}^{\infty} \operatorname{dim}_{\mathbb{Z}_{2}} R L_{p} H_{k}(X)$. We call $\chi_{p}(X)=\sum_{k=2 p}^{\infty}(-1)^{k} \operatorname{dim}_{\mathbb{Z}_{2}} L_{p} H_{k}\left(X ; \mathbb{Z}_{2}\right)$ the $L_{p}$-Euler characteristic of $X$ with $\mathbb{Z}_{2}$-coefficients and $R \chi_{p}(R e X)=\sum_{k=p}^{\infty}(-1)^{k-p} \operatorname{dim}_{\mathbb{Z}_{2}} R L_{p} H_{k}(X)$ the real $L_{p}$-Euler characteristic.

Let $B(p)(X)_{\mathbb{R}}=\sum_{k=0}^{\infty} \operatorname{dim}_{\mathbb{Z}_{2}} \pi_{k}\left(\frac{Z_{p}(X)_{\mathbb{R}}}{2 Z_{p}(X)_{\mathbb{R}}}\right)$.

Theorem 4.6. Suppose $X$ is a real projective variety. If $B(p)(X)$ and $B(p)(X)_{\mathbb{R}}$ are finite, then

(1) $\chi_{p}(X) \equiv R \chi_{p}(\operatorname{Re} X) \bmod 2$,

(2) $B(p)(X) \equiv \beta(p)(X) \bmod 2$.

If in addition $G$ is weakly contractible, then

(3) $B(p)(X) \geq \beta(p)(X)$.

Proof. To simplify the notation, we use the same notation as in Proposition 4.3 but with a different meaning. We use $M_{n}$ to denote the rank of the $n$-th homotopy group of $M$, and $\operatorname{Kerg}_{n}$ and $I m g_{n}$ the rank of the kernel and the rank of the image over $\mathbb{Z}_{2}$ of a homomorphism $g_{n}$ respectively.

From the finiteness assumption on $B(p)(X)$ and $B(p)(X)_{\mathbb{R}}$, we know that $\sum_{n=0}^{\infty} C_{n}$ and $\sum_{n=0}^{\infty} E_{n}$ are finite from the long exact sequence (1) and (2) respectively in Proposition 4.3.

(1) From the first two long exact sequences in Proposition 4.3, we have $\chi_{p}(B)=$ $\chi_{p}(A)+\chi_{p}(C)$ and $\chi_{p}(A)=\chi_{p}(C)+\chi_{p}(E)$, thus $\chi_{p}(B) \equiv \chi_{p}(E) \bmod 2$.

(2) From the long exact sequence 1 , we have $A_{n}=I m c_{n+1}+\operatorname{Im} a_{n}=C_{n+1}-$ $k e r c_{n+1}+B_{n}-I m b_{n}$, and from the long exact sequence (2), we have $A_{n}=$ $I m a_{n}^{\prime}+I m c_{n}^{\prime}=E_{n}-k e r c_{n-1}^{\prime}+c_{n}-k e r c_{n}^{\prime}$. Simplifying the equation and taking sums, we get $\sum B_{n}=\sum E+2 \sum\left(k e r c_{n}-k e r c_{n}^{\prime}\right)$. Thus $B(p)(X) \equiv$ $\beta(p)(X) \bmod 2$.

(3) If $G$ is weakly contractible, then $\pi_{k}(G)=0$ for all $k$. From the long exact sequence (3) and (4), we have $A_{n}=D_{n}$ and $C_{n}=F_{n}$. Since by Theorem 3.9, $D_{n}=E_{n}+F_{n}$, we have $A_{n}=C_{n}+E_{n}$ for all $n$. From the long exact sequence (1), we have $A_{n}=C_{n+1}-\operatorname{kerc}_{n+1}+B_{n}-\operatorname{Imb} b_{n}$. Thus $C_{n}+E_{n} \leq C_{n+1}+B_{n}$. Taking the sum over all $n$, we have $\sum E_{n} \leq \sum B_{n}$.

For zero-cycles, to simplify the notation, we simply write $B(X)=B(0)(X)$, $B(\operatorname{Re} X)=\beta(0)(X), \chi(X)=\chi_{0}(X)$ and $\chi(\operatorname{Re} X)=R \chi_{0}(\operatorname{Re} X)$ which are the standard total Betti numbers and Euler characteristic of $X$ and $\operatorname{Re} X$ in $\mathbb{Z}_{2}$-coefficients. 
Corollary 4.7 (Harnack-Thom Theorem). Let $X$ be a real projective variety. Then

(1) $B(X) \geq \beta(\operatorname{Re} X)$,

(2) $B(X) \equiv \beta(\operatorname{Re} X) \bmod 2$,

(3) $\chi(X) \equiv \chi(\operatorname{Re} X) \bmod 2$.

Proof. For $p=0$, by Proposition 3.4, $Z Q_{0}(X)_{\mathbb{R}}=2 Z_{0}(X)_{\mathbb{R}}$, thus $G=\frac{Z Q_{0}(X)_{\mathbb{R}}}{2 Z_{0}(X)_{\mathbb{R}}}$ is trivial. By the Dold-Thom theorem, $\pi_{k}\left(\frac{Z_{0}(X)}{2 Z_{0}(X)}\right)=H_{k}\left(X ; \mathbb{Z}_{2}\right), \pi_{k}\left(\frac{Z_{0}(X)_{\mathbb{R}}}{2 Z_{0}(X)_{\mathbb{R}}}\right)=$ $H_{k}\left(X / \mathbb{Z}_{2} ; \mathbb{Z}_{2}\right)$, where $X / \mathbb{Z}_{2}$ is the orbit space of $X$ under the action of conjugation. Thus $B(0)(X)$ and $B(0)(X)_{\mathbb{R}}$ are finite. The result now follows from the theorem above.

Suppose that $Y \subset X$ are real projective varieties and $U=X-Y$ is a real quasiprojective variety. Let $A, B, \ldots, G$ be the cycle groups of $X$ defined as above and let $A^{\prime}=\frac{Z_{p}(Y)_{\mathbb{R}}}{2 Z_{p}(Y)_{\mathbb{R}}}, B^{\prime}=\frac{Z_{p}(Y)}{2 Z_{p}(Y)}, C^{\prime}=\frac{Z_{p}(Y)^{a v}}{2 Z_{p}(Y)_{\mathbb{R}}}, D^{\prime}=\frac{Z_{p}(Y)_{\mathbb{R}}}{Z Q_{p}(Y)_{\mathbb{R}}}, E^{\prime}=\frac{Z_{p}(Y)_{\mathbb{R}}}{Z_{p}(Y)^{a v}}$, $F^{\prime}=\frac{Z_{p}(Y)^{a v}}{Z Q_{p}(Y)_{\mathbb{R}}}, G^{\prime}=\frac{Z Q_{p}(Y)_{\mathbb{R}}}{2 Z_{p}(Y)_{\mathbb{R}}}$.

We can check as in Proposition 2.8 of [18] that $T^{\prime}$ is embedded as a closed subgroup of $T$; thus we identify $T^{\prime}$ with its image in $T$ for any group $T$ above. To simplify the notation, we will use $\left(T^{\prime}\right)$ to mean the image of $T^{\prime}$ in $T$.

Lemma 4.8. We have two short exact sequences of topological abelian groups:

(1) $0 \rightarrow \frac{A}{A^{\prime}} \stackrel{i}{\longrightarrow} \frac{B}{B^{\prime}} \stackrel{1+c_{*}}{\longrightarrow} \frac{C}{C^{\prime}} \rightarrow 0$,

(2) $0 \rightarrow \frac{C}{C^{\prime}} \rightarrow \frac{A}{A^{\prime}} \rightarrow \frac{E}{E^{\prime}} \rightarrow 0$

and an equation

$$
\frac{D}{D^{\prime}}=\frac{E}{E^{\prime}} \times \frac{F}{F^{\prime}}
$$

Proof. We prove that the first sequence is exact and a similar argument works for the second one. Injectivity: let $a \in Z_{p}(X)_{\mathbb{R}}$ and $a+2 Z_{p}(X)+\left(\frac{Z_{p}(Y)}{2 Z_{p}(Y)}\right) \in$ $\left(\frac{Z_{p}(Y)}{2 Z_{p}(Y)}\right)$; then $a=2 b+c$ where $b \in Z_{p}(X), c \in Z_{p}(Y)$, and we may assume $b, c$ have no common components and the conjugation of each component of $c$ is not a component of $b$. Since $a$ is real, $b, c$ are real hence $a$ is 0 in $\frac{A}{A^{\prime}}$. It is trivial that the image of $i$ is contained in the kernel of $1+c_{*}$ and the map $1+c_{*}$ is surjective. Suppose that for $a \in Z_{p}(X), a+\bar{a}+2 Z_{p}(X)_{\mathbb{R}}+\left(\frac{Z_{p}(Y)^{a v}}{2 Z_{p}(Y)_{\mathbb{R}}}\right) \in\left(\frac{Z_{p}(Y)^{a v}}{2 Z_{p}(Y)_{\mathbb{R}}}\right)$. Since $a+\bar{a}=2 R P(a)+2 A P(a)+I P(a)+I P(\bar{a})$, we have $I P(a)+I P(\bar{a}) \in$ $Z_{p}(Y)^{a v}$. From the definition of $I P$, we see that $I P(a) \in Z_{p}(Y)$. Therefore $i$ sends $R P(a)+A P(a)+2 Z_{p}(X)_{\mathbb{R}}+\left(\frac{Z_{p}(Y)}{2 Z_{p}(Y)}\right)$ to $a+2 Z_{p}(X)_{\mathbb{R}}+\left(\frac{Z_{p}(Y)^{a v}}{2 Z_{p}(Y)_{\mathbb{R}}}\right)$ which implies that the sequence is exact.

By a similar calculation as in Theorem 4.6, we get

Theorem 4.9. Suppose that $U$ is a real quasiprojective variety. If $B(p)(U)$ and $B(p)(U)_{\mathbb{R}}$ are finite, then

(1) $\chi_{p}(U) \equiv R \chi_{p}(\operatorname{Re} U) \bmod 2$,

(2) $B(p)(U) \equiv \beta(p)(U) \bmod 2$.

If in addition $\frac{G}{G^{\prime}}$ is weakly contractible, then

(3) $B(p)(U) \geq \beta(p)(U)$. 
Corollary 4.10 (Harnack-Thom Theorem for real quasiprojective varieties). Suppose that $U$ is a real quasiprojective variety. Let $B^{B M}(U), \beta^{B M}(\operatorname{Re} U), \chi^{B M}(U)$, $\chi^{B M}(R e U)$ denote the total Betti number and the Euler characteristic of $U$ and $R e(U)$ respectively in the Borel-Moore homology with $\mathbb{Z}_{2}$-coefficients. Then

(1) $\beta^{B M}(\operatorname{Re} U) \leq B^{B M}(U)$,

(2) $B^{B M}(U) \equiv \bar{\beta}^{B M}(\operatorname{Re} U) \bmod 2$,

(3) $\chi^{B M}(U) \equiv \chi^{B M}(\operatorname{Re} U) \bmod 2$.

Proof. By the Dold-Thom theorem of $\mathbb{Z}_{2}$-coefficients, we have

$$
\pi_{k}\left(\frac{Z_{0}(U)}{2 Z_{0}(U)}\right)=H_{k}^{B M}\left(U ; \mathbb{Z}_{2}\right) \text { and } \pi_{k} R_{0}(U)=H_{k}^{B M}\left(R e U ; \mathbb{Z}_{2}\right) .
$$

For zero-cycles, the equation $\frac{D}{D^{\prime}}=\frac{E}{E^{\prime}} \times \frac{F}{F^{\prime}}$ becomes $\frac{A}{A^{\prime}}=\frac{E}{E^{\prime}} \times \frac{C}{C^{\prime}}$. Now Theorem 4.9 suffices to finish the proof.

Example 4.11. From the Splitting Principle in reduced real morphic cohomology (see Theorem 5.4 in [18]) and the Duality Theorem between reduced real morphic cohomology and reduced real Lawson homology (see Theorem 6.2 in [18]), we get

$$
R_{1}\left(\mathbb{P}^{1} \times \mathbb{P}^{1}\right)=R_{0}\left(\mathbb{P}^{1}\right) \times R_{1}\left(\mathbb{P}^{1}\right) .
$$

Thus $\pi_{0}\left(R_{1}\left(\mathbb{P}^{1} \times \mathbb{P}^{1}\right)\right)=\mathbb{Z}_{2} \oplus \mathbb{Z}_{2}, \pi_{1}\left(R_{1}\left(\mathbb{P}^{1} \times \mathbb{P}^{1}\right)\right)=\mathbb{Z}_{2} . \quad R \chi_{1}\left(\mathbb{R P}^{1} \times \mathbb{R P}^{1}\right)=$ $1, \beta(1)\left(\mathbb{P}^{1} \times \mathbb{P}^{1}\right)=3$. From the Splitting Principle in morphic cohomology (see Theorem 2.10 in [6]) and the Duality Theorem between morphic cohomology and Lawson homology, we have

$$
Z_{1}\left(\mathbb{P}^{1} \times \mathbb{P}^{1}\right)=Z_{0}\left(\mathbb{P}^{1}\right) \times Z_{1}\left(\mathbb{P}^{1}\right),
$$

which gives $\pi_{0}\left(\frac{Z_{1}\left(\mathbb{P}^{1} \times \mathbb{P}^{1}\right)}{2 Z_{1}\left(\mathbb{P}^{1} \times \mathbb{P}^{1}\right)}\right)=\mathbb{Z}_{2} \oplus \mathbb{Z}_{2}, \pi_{2}\left(\frac{Z_{1}\left(\mathbb{P}^{1} \times \mathbb{P}^{1}\right)}{2 Z_{1}\left(\mathbb{P}^{1} \times \mathbb{P}^{1}\right)}\right)=\mathbb{Z}_{2}$. Thus $\chi_{1}\left(\mathbb{P}^{1} \times \mathbb{P}^{1}\right)=$ $B(1)\left(\mathbb{P}^{1} \times \mathbb{P}^{1}\right)=3$.

Example 4.12. Consider $\mathbb{C}^{n}=\mathbb{P}^{n}-\mathbb{P}^{n-1}$. From the Lawson suspension theorem, we are able to get $\frac{Z_{p}\left(\mathbb{C}^{n}\right)}{2 Z_{p}\left(\mathbb{C}^{n}\right)}=K\left(\mathbb{Z}_{2}, 2(n-p)\right), R_{p}\left(\mathbb{C}^{n}\right)=K\left(\mathbb{Z}_{2}, n-p\right)$. Therefore we have

$$
\begin{gathered}
L_{p} H_{k}\left(\mathbb{C}^{n} ; \mathbb{Z}_{2}\right)= \begin{cases}\mathbb{Z}_{2}, & \text { if } k=2 n, \\
0, & \text { otherwise, }\end{cases} \\
R L_{p} H_{k}\left(\mathbb{C}^{n}\right)= \begin{cases}\mathbb{Z}_{2}, & \text { if } k=n, \\
0, & \text { otherwise },\end{cases}
\end{gathered}
$$

and then $\chi_{p}\left(\mathbb{C}^{n}\right)=1, R \chi_{p}\left(\mathbb{R}^{n}\right)=(-1)^{n-p}, B(p)\left(\mathbb{A}^{n}\right)=\beta(p)\left(\mathbb{A}^{n}\right)=1$.

The reduced real Lawson homology groups of a variety naturally depend on its real structure. Two real projective varieties may be isomorphic as complex projective varieties, but they may not be isomorphic as real projective varieties. Thus reduced real Lawson homology groups may be used to distinguish two real projective varieties.

Example 4.13. Let $X$ be the smooth quadric defined by the equation $x^{2}+y^{2}+z^{2}=$ 0 in $\mathbb{P}^{2}$. The variety $X$ is complex algebraically isomorphic to $\mathbb{P}^{1}$ but not real algebraically isomorphic to $\mathbb{P}^{1}$ since $X$ has no real point. Therefore all the reduced real Lawson homology groups of zero-cycles on $X$ are trivial, but $B(0)(X)=\chi_{0}(X)=2$. 


\section{A construction of Weil}

Throughout this section, $X$ is a nonsingular projective variety of dimension $m$. Let $G$ be the fundamental group of $X$ and $G^{\prime}$ be the commutator-group of $G$. Then $H=G / G^{\prime}=H_{1}(X, \mathbb{Z})$ is the first homology group of $X$ with integral coefficients.

Definition 5.1. Let $p:(\hat{X}, y) \longrightarrow(X, x)$ be a covering map where $\hat{X}, X$ are complex manifolds. The covering is said to be abelian if $p_{*} \pi_{1}(\hat{X}, y)=G^{\prime}$.

For an abelian covering, the group of deck transformations is isomorphic to $H$. Every element $\sigma \in H$ determines an automorphism of $\hat{X}$, transforming each point $\hat{s}$ of $\hat{X}$ into a point $\sigma(\hat{s})$ lying over the same point $s$ in $X$.

Let $\epsilon$ be a group homomorphism of $H$ into the multiplicative group $\mathbb{C}^{*}$. A multiplicative function on $\hat{X}$ with the multiplicator-set $\epsilon$ is a nonidentically zero meromorphic function $\phi$ on $\hat{X}$ such that $\phi(\sigma \hat{s})=\phi(\hat{s}) \epsilon(\sigma)$ for all $\hat{s} \in \hat{X}, \sigma \in H$.

Let $T$ be the torsion subgroup of $H$. A multiplicator-set which is 1 on $T$ is called special. Let $\Theta_{X}$ be the group of all special multiplicator-sets $\epsilon$ where $|\epsilon(\sigma)|=1$ for all $\sigma \in H$. A divisor $Z$ on $X$ is defined by a meromorphic multiplicative function $\phi$ on $\hat{X}$, as explained in Page 873 of [20], by taking the zero locus of $\phi$, and the multiplicator-set of $\phi$ is special if and only if $Z$ is algebraically equivalent to zero. It is proved in [20] that $\Theta_{X}$ is an abelian variety for a nonsingular projective variety $X$ and the real dimension of $\Theta_{X}$ is equal to the rank of $H$.

Definition 5.2. For $X$ a nonsingular projective variety, the abelian variety $\Theta_{X}$ is called the Picard variety of $X$.

Let $Z_{m-1}(X)^{\text {alg }}$ be the group of divisors on $X$ which are algebraically equivalent to zero.

Definition 5.3. (Weil's construction) Define a group homomorphism

$$
w: Z_{m-1}(X)^{\text {alg }} \longrightarrow \Theta_{X}
$$

by

$$
w(Z)=\epsilon
$$

where $\epsilon \in \Theta_{X}$ is the special multiplicator-set of $\phi$ and $Z$ is the divisor defined by $\phi$.

For the reader's convenience, we recall a definition from [20].

Definition 5.4. An analytic family of divisors on a nonsingular projective variety $X$ parametrized by a nonsingular projective variety $S$ is an algebraic cycle $V$ on $S \times X$ such that $V_{s}:=\operatorname{Pr}_{*}(V \bullet(s \times X))$ is a divisor on $X$ (where $\operatorname{Pr}: S \times X \longrightarrow X$ is the projection and $\bullet$ is the intersection product). A mapping $f: Z_{m-1}(X)^{\text {alg }} \longrightarrow$ $\Theta_{X}$ is said to be analytic if for any analytic family of divisors algebraically equivalent to zero on $X$, parametrized by $S$, the map $f \circ \lambda: S \longrightarrow \Theta_{X}$ is an analytic map where $\lambda: S \longrightarrow Z_{m-1}(X)^{\text {alg }}$ is the parametrization.

Let $Z_{m-1}(X)^{\text {lin }}$ be the group of divisors on $X$ which are linearly equivalent to zero. The following is the "Main Theorem" in Weil's paper [20].

Theorem 5.5. The surjective group homomorphism $w: Z_{m-1}(X)^{\text {alg }} \longrightarrow \Theta_{X}$ in Weil's construction is analytic, and the kernel of $w$ is $Z_{m-1}(X)^{l i n}$. There is a bijective parametrization $\Lambda: \Theta_{X} \longrightarrow \frac{Z_{m-1}(X)^{\text {alg }}}{Z_{m-1}(X)^{l i n}}$. 
The main result we need is that the topology on $\Theta_{X}$ is actually the same as the topology on $\frac{Z_{m-1}(X)^{\text {alg }}}{Z_{m-1}(X)^{l i n}}$.

Corollary 5.6. The map $w: Z_{m-1}(X)^{\text {alg }} \longrightarrow \Theta_{X}$ in Weil's construction is continuous and therefore it induces a topological group isomorphism

$$
\widetilde{w}: \frac{Z_{m-1}(X)^{\text {alg }}}{Z_{m-1}(X)^{l i n}} \longrightarrow \Theta_{X}
$$

Proof. We may form a topology on $Z_{m-1}(X)^{\text {alg }}$ by declaring that a set $U \subset$ $Z_{m-1}(X)^{\text {alg }}$ is open if and only for all parametrizations $\lambda: S \longrightarrow Z_{m-1}(X)^{\text {alg }}$, $\lambda^{-1}(U)$ is open. By Theorem 2.16 in [13], this topology coincides with the Chow topology. Combining this with Theorem 5.5, we have that $w$ is a continuous map. From Weil's construction, $\widetilde{w} \circ \Lambda=$ the identity map. Since $\Theta_{X}$ is compact, $\Lambda$ is a topological group isomorphism, which implies that $\widetilde{w}$ is a topological group isomorphism.

Definition 5.7. We say that a complex manifold $(M,-)$ with a map $-: M \longrightarrow M$ is real if the map - is an antiholomorphic map and $-{ }^{2}=$ the identity map. The map - is called the conjugation of $M$.

All nonsingular projective varieties defined by real polynomials in $\mathbb{P}^{n}$ have a natural conjugation which is induced by the standard conjugation of $\mathbb{P}^{n}$.

Definition 5.8. If $\hat{X}$ and $X$ are real complex manifolds and the covering map $p: \hat{X} \longrightarrow X$ satisfies $p(\bar{z})=\overline{p(z)}$ for all $z \in \hat{X}$, then the covering is said to be real.

Lemma 5.9. Suppose that $p:(\hat{X}, y) \longrightarrow(X, x)$ is a covering map where $\hat{X}$ is a complex manifold and $X$ is real complex manifold. Then the conjugation on $X$ induces a conjugation on $\hat{X}$ such that the covering is real.

Proof. For each point $t \in X$, take a small connected open neighborhood $U_{t}$ and a biholomorphic local trivialization $\phi_{t}: p^{-1}\left(U_{t}\right) \longrightarrow U_{t} \times F$ where $F$ is the fibre which is discrete. We may take $U_{t}$ small enough and make $U_{\bar{t}}=\overline{U_{t}}$ for all $t$. We define a conjugation on $U_{t} \times F$ by $\overline{(w, b)}=(\bar{w}, b)$. Suppose that $z$ is a point in the fibre over $U_{t}$; define $\bar{z}=\phi_{\bar{t}}^{-1} \overline{\phi_{t}(z)}$. It is not difficult to check that $\overline{\bar{z}}=z$. The map $\phi_{\bar{t}} \circ \phi_{\bar{s}}^{-1}: \overline{U_{t} \cap U_{s}} \times F \longrightarrow \overline{U_{t} \cap U_{s}} \times F$ is given by $(w, b) \longrightarrow(w, g b)$ where $g$ is a bijection from $F$ to $F$. Thus $\phi_{\bar{t}}^{-1} \circ \phi_{\bar{s}}^{-1}$ is real, i.e., $\phi_{\bar{t}}^{-1} \circ \phi_{\bar{s}}^{-1} \overline{(w, b)}=\overline{\phi_{\bar{t}}^{-1} \circ \phi_{\bar{s}}^{-1}(w, b)}$. It is then easy to verify that $\phi_{\bar{t}}^{-1} \overline{\phi_{t}(z)}=\phi_{\bar{s}}^{-1} \overline{\phi_{s}(z)}$ in the overlap of $U_{t}$ and $U_{s}$. So $\bar{z}$ is well defined. Since $U_{t} \times F$ for any $t$, the map sending $z$ to $\bar{z}$ is antiholomorphic, thus the map we just defined is a conjugation on $\hat{X}$.

We remark that this conjugation depends on the choice of local trivializations, for instance in the case of trivial covering space $\hat{X}=X \times F$.

Definition 5.10. Suppose that $p:(\hat{X}, \hat{x}) \longrightarrow(X, x)$ is a real abelian covering and $\sigma$ is a deck transformation induced by a loop $[f] \in \pi_{1}(X, x)$. Let $\gamma$ be a path from $x$ to $\bar{x}$ and let $g=\gamma^{-1} * \bar{f} * \gamma$ be the loop at $x$, defined by traveling from $x$ to $\bar{x}$ along $\gamma$, going around $\bar{x}$ along the conjugation of $f$ and then traveling back to $x$ along $\gamma$ in the opposite direction. Let $\overline{\bar{\sigma}}$ be the deck transformation defined by $g$. If we take another path $\gamma^{\prime}$ from $x$ to $\bar{x}$ and let $g^{\prime}=\gamma^{\prime-1} * \bar{f} * \gamma^{\prime}$, then it is easy 
to show that $g^{\prime} g^{-1}$ is an element in the commutator group, thus $g^{\prime}$ defines a same deck transformation as $g$ does. We can check that

$$
\overline{\bar{\sigma}}(z)=\overline{\sigma(\bar{z})}
$$

for all $z \in \hat{X}$. We say that $\sigma$ is real if $\sigma=\overline{\bar{\sigma}}$.

From the theory of covering spaces, we know that $\sigma$ is real if and only $[f]=[g]$ in $\pi_{1}(X, x) / p_{*}\left(\pi_{1}(\hat{X}, \hat{x})\right)$.

If $X$ is a nonsingular real projective manifold and $\phi$ is a multiplicative function on $\hat{X}$, define $\overline{\bar{\phi}}(z):=\overline{\phi(\bar{z})}$ which is also a multiplicative function on $\hat{X}$.

Let $\epsilon \in \Theta_{X}$ be a special multiplicator-set and define $\bar{\epsilon}(\sigma):=\overline{\epsilon(\overline{\bar{\sigma}})}$. If $\epsilon=\bar{\epsilon}$, then we say that $\epsilon$ is real. The map $\epsilon \longrightarrow \bar{\epsilon}$ induces a conjugation on $\Theta_{X}$ which makes $\Theta_{X}$ a real complex manifold.

Lemma 5.11. Suppose that $p:(\hat{X}, \hat{x}) \longrightarrow(X, x)$ is a real abelian covering and $X$ is a real projective manifold. Let $Z$ be a divisor of $X$. If $\phi$ is a multiplicative function defining $Z$, with multiplicator-set $\epsilon$, then $\overline{\bar{\phi}}$ is a multiplicative function defining $\bar{Z}$, with multiplicator-set $\bar{\epsilon}$.

Proof. $\overline{\bar{\phi}}(\sigma(y))=\overline{\phi(\overline{\sigma(y)})}=\overline{\phi(\overline{\bar{\sigma}}(\bar{y}))}=\overline{\phi(\bar{y}) \epsilon(\overline{\bar{\sigma}})}=\overline{\bar{\phi}}(y) \bar{\epsilon}(\sigma)$.

It was shown by Weil in [20] that a divisor $Z$ on $X$ is linearly equivalent to 0 if and only if for a multiplicative function $\phi$ defining $Z$, the special multiplicator-set $\epsilon$ of $\phi$ is 1 . Therefore, since $\bar{Z}$ is defined by $\overline{\bar{\phi}}$ with multiplicator-set $\bar{\epsilon}$, this implies that $\bar{Z}$ is also linearly equivalent to 0 . So the conjugation on $Z_{m-1}(X)^{\text {alg }}$ passes to $\frac{Z_{m-1}(X)^{\text {alg }}}{Z_{m-1}(X)^{l i n}}$.

By Lemma 5.11, it is clear that we have the following result.

Theorem 5.12. Suppose that $p:(\hat{X}, \hat{x}) \longrightarrow(X, x)$ is a real abelian covering and $X$ is a real projective manifold. Then the map $\widetilde{w}: \frac{Z_{m-1}(X)^{a l g}}{Z_{m-1}(X)^{l i n}} \longrightarrow \Theta_{X}$ in Weil's construction is real, i.e., $\widetilde{w}(\overline{[Z]})=\overline{w(Z)}$ for all $Z \in Z_{m-1}(X)^{\text {alg }}$.

Definition 5.13. Suppose that $p:(\hat{X}, \hat{x}) \longrightarrow(X, x)$ is a real abelian covering and $X$ is a real projective manifold. We say that $X$ is real symmetric if all the deck transformations of $X$ are real.

Proposition 5.14. Suppose that $p:(\hat{X}, \hat{x}) \longrightarrow(X, x)$ is a real abelian covering and $X$ is real symmetric. If a divisor $Z$ is algebraically equivalent to 0 , then the averaged divisor $Z+\bar{Z}$ is linearly equivalent to 0 .

Proof. Let $\phi$ be a meromorphic multiplicative function defining $Z$, with special multiplicator-set $\epsilon$. Since all deck transformations are real, we have $\bar{\epsilon}(\sigma)=\overline{\epsilon(\sigma)}$ for all $\sigma \in H$. Then $\epsilon(\sigma) \bar{\epsilon}(\sigma)=1$ which implies that $Z+\bar{Z}$ is linearly equivalent to 0 .

Corollary 5.15. A projective curve $X$ is not real symmetric if the genus $g$ of $X$ is greater than 0 .

Proof. Let $p \in X$ and $D=p+\bar{p}$. For a divisor $E$ on $X$, let $L(E)$ be the dimension of $H^{0}(X,[E])$ where $[E]$ is the line bundle associated to $E$, and let $|E|$ be the linear system associated to $E$. If $g=1$, by Riemann-Roch theorem, we have $L(D)=2$. If $g>1, L(D) \geq 1$ and $L(K-D) \geq 1$ where $K$ is a canonical divisor 
on $X$, then by Clifford's theorem, $L(D) \leq 2$. Assume that for every $q \in X, q+\bar{q}$ is linearly equivalently to $D$. Then $\operatorname{dim}|D|=L(D)-1=1$. Consider the set $\mathscr{C}_{0,2}(X)=S P^{2}(X)$ of effective divisors of degree 2 where $S P^{2}(X)$ is the 2-fold symmetric product of $X$. We have $\mathscr{C}_{0,2}(X)_{\mathbb{R}}=S P^{2}(X)_{\mathbb{R}}=\{q+\bar{q} \mid q \in X\}$, and by the assumption we have $\mathscr{C}_{0,2}(X)_{\mathbb{R}} \subset|D|=\mathbb{P}^{1}$. Since the map $X \longrightarrow S P^{2}(X)_{\mathbb{R}}$ defined by $a \longrightarrow a+\bar{a}$ is a homeomorphism, it gives an embedding of $X$ into $\mathbb{P}^{1}$ which is impossible. Therefore, there exists $q \in X$ such that $q+\bar{q} \notin|D|$. Since $p-q$ is algebraically equivalent to zero but $(p-q)+\overline{(p-q)}=(p+\bar{p})-(q+\bar{q})$ is not linearly equivalent to zero, this contradicts the conclusion of Proposition 5.14. Hence, $X$ is not real symmetric.

Lemma 5.16. If $D$ is a real divisor which is linearly equivalent to 0 , then there is a real rational function $F$ such that $D=(F)$, the divisor defined by $F$.

Proof. Let $D=D_{1}-D_{2}$ where $D_{1}$ and $D_{2}$ are effective real divisors. Since $D$ is linearly equivalent to zero, there exists a rational function $F=\frac{f}{g}$ such that $D=$ $(F)$. Suppose that $(f)=D_{1}+D_{3},(g)=D_{2}+D_{3}$. Since $D_{3}+\bar{D}_{3}$ is a real divisor, we can take a real homogeneous polynomial $h$ such that $(h)=D_{3}+\bar{D}_{3}+D_{4}$. We show that we can find a real homogeneous polynomial which defines the divisor $(f h)$. By splitting the coefficients of $f h$ into the form $x+i y$, we may write $f h=G+i H$ where $G, H$ are real homogeneous polynomials. If $G$ is zero, then $(f h)=(i H)=(H)$. If $G$ is not zero, then the degree of $G$ equals the degree of $f h$. If $f(z) h(z)=0$, then $f(\bar{z}) h(\bar{z})=0$. We have $G(z)+i H(z)=0$ and $G(\bar{z})+i H(\bar{z})=G(z)-i H(z)=0$. This implies that $G(z)=0$. Thus $(G)=(f h)=D_{1}+2 D_{3}+\bar{D}_{3}+D_{4}$ is a real divisor. Similarly, we can find a real homogenous polynomial $G^{\prime}$ such that $\left(G^{\prime}\right)=(g h)=D_{2}+2 D_{3}+\bar{D}_{3}+D_{4}$. Therefore, $D=(f h)-(g h)=\left(\frac{G}{G^{\prime}}\right)$.

Proposition 5.17. If $D$ is a real divisor which is linearly equivalent to 0 , then $D$ is in the 0-component of $Z_{m-1}(X)_{\mathbb{R}}$.

Proof. Let $(x, y) \in \mathbb{C}^{2} \backslash\{0\}, D=(F)$ where $F$ is a real rational function. Let $V_{x, y}$ be the divisor defined by $x+y F$. We have $V_{0,1}=D$ and $V_{1,0}=0$. Let $\gamma:[0,1] \longrightarrow \mathbb{C}^{2} \backslash\{0\}$ be the path given by $\gamma(t)=(t, 1-t)$. Then each $V_{\gamma(t)}$ is real, and this gives a path in $Z_{m-1}(X)_{\mathbb{R}}$ joining $D$ and 0 .

It follows from this result that we do not have to distinguish between real and complex linear equivalence in $Z_{m-1}(X)_{\mathbb{R}}$.

Denote $Z_{m-1}(X)_{\mathbb{R}}^{0}$ to be the 0 -component of $Z_{m-1}(X)_{\mathbb{R}}$ and $Z_{m-1}(X)_{\mathbb{R}}^{\text {lin }}=\{a \in$ $Z_{m-1}(X)_{\mathbb{R}} \mid a$ is linearly equivalent to 0$\}$.

Corollary 5.18. Suppose that $X$ is a real nonsingular projective variety of dimension $m$. We have the following inclusions:

$$
Z_{m-1}(X)_{\mathbb{R}}^{l i n} \subset Z_{m-1}(X)_{\mathbb{R}}^{0} \subset Z_{m-1}(X)^{\text {alg }} .
$$

Definition 5.19. Let $R_{m-1}(X)^{0}$ be the connected component of $R_{m-1}(X)$ containing 0 and $R_{m-1}(X)^{l i n}=\left\{a+Z_{m-1}(X)^{a v} \mid a\right.$ is linearly equivalent to some $b$, where $\left.b \in Z_{m-1}(X)^{a v}\right\}$.

It is easy to check the following result. 
Lemma 5.20. Suppose that $X$ is a nonsingular real projective variety of dimension $m$. Then

$$
\begin{aligned}
R_{m-1}(X)^{0} & =\frac{Z_{m-1}(X)_{\mathbb{R}}^{0}}{Z_{m-1}(X)_{\mathbb{R}}^{0} \cap Z_{m-1}(X)^{a v}}, \\
R_{m-1}(X)^{l i n} & =\frac{Z_{m-1}(X)_{\mathbb{R}}^{l i n}}{Z_{m-1}(X)_{\mathbb{R}}^{l i n} \cap Z_{m-1}(X)^{a v}} .
\end{aligned}
$$

The inclusion map $R_{m-1}(X)^{\text {lin }} \hookrightarrow R_{m-1}(X)^{0}$ is a closed embedding. We will abusively denote the image of $R_{m-1}(X)^{l i n}$ in $R_{m-1}(X)^{0}$ by $R_{m-1}(X)^{l i n}$.

Let $P i c^{0}(X)$ be the group of holomorphic line bundles on $X$ whose first Chern class is zero. There is an isomorphism

$$
u: \frac{Z_{m-1}(X)^{a l g}}{Z_{m-1}^{\operatorname{lin}}(X)} \longrightarrow \operatorname{Pic}^{0}(X)
$$

where $u$ maps a divisor $Z$ to the line bundle associated to $Z$. We give a topology on $\operatorname{Pic}^{0}(X)$ by making $u$ a homeomorphism. For $L \in P i c^{0}(X), L=[c]$ for some $c \in Z_{m-1}(X)^{a l g}$. We define $\bar{L}=[\bar{c}]$. Then the map $u$ is real. We have the following commutative diagram and each map is a real topological group isomorphism:

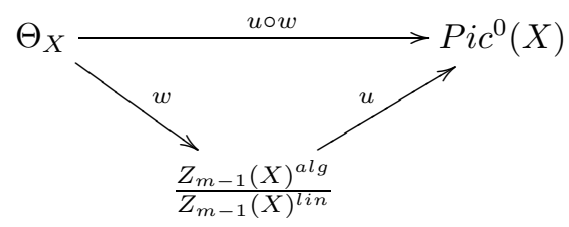

Definition 5.21. We say that a holomorphic line bundle $L$ on a nonsingular projective variety $X$ is real if $L$ is the line bundle associated to some real divisor, and $L$ is averaged if $L$ is the line bundle associated to some averaged divisor. Denote $\operatorname{Pic}^{0}(X)_{\mathbb{R}}$ to be the 0-component of real line bundles and $\operatorname{Pic}^{0}(X)^{a v}$ to be the 0 component of averaged line bundles. We define the reduced real Picard group of $X$ to be

$$
\operatorname{RPic}^{0}(X)=\frac{\operatorname{Pic}^{0}(X)_{\mathbb{R}}}{\operatorname{Pic}^{0}(X)^{a v}}
$$

which is a topological abelian group.

The real isomorphism $u$ gives us the following result.

Theorem 5.22. For a nonsingular real projective variety $X$ of dimension $m$, we have a topological group isomorphism:

$$
\widetilde{u}: \frac{R_{m-1}(X)^{0}}{R_{m-1}(X)^{\operatorname{lin}}} \longrightarrow \operatorname{RPic}^{0}(X)
$$

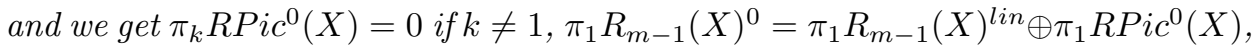
and $\pi_{i} R_{m-1}(X)^{0}=\pi_{i} R_{m-1}(X)^{\text {lin }}$ for $i \neq 1$.

Proof. Since $P i c^{0}(X)_{\mathbb{R}}, P i c^{0}(X)^{a v}$ are closed subgroups of $P i c^{0}(X)$, they are real tori. The results follow from the homotopy sequences induced by the two exact 
sequences:

$$
\begin{aligned}
& 0 \longrightarrow \operatorname{Pic}^{0}(X)^{a v} \longrightarrow \operatorname{Pic}^{0}(X)_{\mathbb{R}} \longrightarrow \operatorname{RPic}^{0}(X) \longrightarrow 0 \\
& 0 \longrightarrow R_{m-1}(X)^{l i n} \longrightarrow R_{m-1}(X)^{0} \longrightarrow \operatorname{RPic}^{0}(X) \longrightarrow 0 .
\end{aligned}
$$

We may as well define the reduced real Picard group to be

$$
\operatorname{RPic}^{0}(X)=\frac{\Theta_{X, \mathbb{R}}^{0}}{\Theta_{X, a v}^{0}}
$$

where $\Theta_{X, \mathbb{R}}^{0}$ is the 0-component of the group of multiplicator-sets in $\Theta_{X}$ which are invariant under the conjugation of $\Theta_{X}$, and $\Theta_{X, a v}$ is the 0-component of the group consisting of multiplicator-sets of the form $\epsilon \bar{\epsilon}$ for $\epsilon \in \Theta_{X}$. From the commutative diagram above it is easy to see that these two topological groups are isomorphic.

Corollary 5.23. If a nonsingular real projective variety $X$ of dimension $m$ is real symmetric, then $R P i c^{0}(X)$ is the trivial group and therefore $R_{m-1}^{0}(X)=R_{m-1}^{\text {lin }}(X)$.

Proof. Let $\epsilon: H_{1}(X, \mathbb{Z}) \longrightarrow S^{1}$ be a real special multiplicator-set in $\Theta_{X}$. If $X$ is real symmetric, then $\overline{\epsilon(\sigma)}=\epsilon(\sigma)$ for all $\sigma \in H_{1}(X, \mathbb{Z})$ which implies that $\epsilon(\sigma)=1$ or -1 . Hence $\Theta_{X, \mathbb{R}}$ is discrete and therefore $\Theta_{X, \mathbb{R}}^{0}$ is trivial which implies that $\operatorname{RP}_{i c}^{0}(X)$ is trivial and hence $R_{m-1}^{0}(X)=R_{m-1}^{l i n}(X)$.

\section{EXAMPLES}

In this section we are going to compute the reduced real Lawson homology groups of divisors.

Let us recall a computation done by Friedlander in [3], Theorem 4.6.

Theorem 6.1. Suppose that $X$ is a nonsingular projective variety of dimension $m$. Then

$$
\pi_{k} Z_{m-1}(X)= \begin{cases}N S(X), & \text { if } k=0 \\ \pi_{1} \operatorname{Pic}^{0}(X), & \text { if } k=1 \\ \mathbb{Z}, & \text { if } k=2 \\ 0, & \text { otherwise }\end{cases}
$$

We make a similar calculation for the real case by the method developed in this paper.

Proposition 6.2. Suppose that $X$ is a nonsingular real projective variety of dimension $m$. Then

$$
\pi_{k} Z_{m-1}(X)_{\mathbb{R}}= \begin{cases}N S(X)_{\mathbb{R}}, & \text { if } k=0 \\ \mathbb{Z}_{2} \oplus \pi_{1} P i c^{0}(X)_{\mathbb{R}}, & \text { if } k=1 \\ 0, & \text { otherwise }\end{cases}
$$

where $N S(X)_{\mathbb{R}}$ is the real Neron-Severi group which is defined to be $\pi_{0} Z_{m-1}(X)_{\mathbb{R}}$. 
Proof. Suppose that $X \subset \mathbb{P}^{n}$. Let

$$
K[X]_{\mathbb{R}}=\frac{\mathbb{R}\left[z_{0}, \ldots, z_{n}\right]}{I_{\mathbb{R}}(X)}
$$

be the real coordinate ring of $X$ where $I_{\mathbb{R}}(X) \subset \mathbb{R}\left[z_{0}, \ldots, z_{n}\right]$ is the ideal of real polynomials vanishing over $X$. Let $K[X]_{\mathbb{R}}=\bigoplus_{k=0}^{\infty} I_{k}$ where $I_{k}$ is the real vector space generated by homogeneous polynomials of degree $k$ of $X$. Define

$$
K_{d}=\left\{\left(\frac{f}{g}\right) \mid f, g \in I_{d}\right\}
$$

and by Lemma 5.16, we have a filtration

$$
\cdots \subset K_{d} \subset K_{d+1} \subset \cdots=Z_{m-1}(X)_{\mathbb{R}}^{\operatorname{lin}} .
$$

Let $I=\lim _{d \rightarrow \infty} \mathbb{P} I_{d} \times \mathbb{P} I_{d}, \Delta_{d}=$ diagonal of $\mathbb{P} I_{d} \times \mathbb{P} I_{d}$, and $\Delta=\lim _{d \rightarrow \infty} \Delta_{d}$ where $\mathbb{P} I_{d}$ is the real projectivisation of $I_{d}$; thus $I \cong K\left(\mathbb{Z}_{2} \oplus \mathbb{Z}_{2}, 1\right), \Delta \cong K\left(\mathbb{Z}_{2}, 1\right)$. For $\left(f_{1}, g_{1}\right) \in \mathbb{P} I_{d_{1}} \times \mathbb{P} I_{d_{1}},\left(f_{2}, g_{2}\right) \in \mathbb{P} I_{d_{2}} \times \mathbb{P} I_{d_{2}}$, we define $\left(f_{1}, g_{1}\right) \cdot\left(f_{2}, g_{2}\right):=$ $\left(f_{1} f_{2}, g_{1} g_{2}\right)$ which induces a monoid structure on $I$ and $\Delta$.

Let $\widetilde{I}, \widetilde{\Delta}$ be the naive group completions of $I$ and $\Delta$ respectively. Since all $\Delta_{d}, \mathbb{P} I_{d}$ are compact $\mathrm{CW}$-complexes, the monoids $\Delta$ and $I$ are free, strongly properly $c$ graded (see [14] for the definitions). By Theorem 4.4' of [14], $\widetilde{I}, \widetilde{\Delta}$ are homotopy equivalent to their homotopy theoretic group completions respectively. Hence $\pi_{k} \widetilde{I}=$ $\pi_{k} I$, and $\pi_{k} \widetilde{\Delta}=\pi_{k} \Delta$ for $k>0$ and $\pi_{0} \widetilde{I}=\pi_{0} \widetilde{\Delta}=\mathbb{Z}$. Since $(I, \Delta)$ is a properly $c$-filtered free pair of monoids, by Theorem 5.2 of [14], we have a fibration

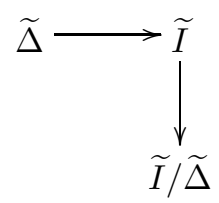

which implies that $\widetilde{I} / \widetilde{\Delta} \cong K\left(\mathbb{Z}_{2}, 1\right)$.

There is a surjective monoid homomorphism $\phi: I \longrightarrow Z_{m-1}(X)_{\mathbb{R}}^{\text {lin }}$ defined by $\phi(f, g)=\left(\frac{f}{g}\right)$. We extend it to a group homomorphism $\widetilde{\phi}: \widetilde{I} \rightarrow Z_{m-1}(X)_{\mathbb{R}}^{\text {lin }}$ by defining $\widetilde{\phi}\left((f, g)-\left(f^{\prime}, g^{\prime}\right)\right)=\phi(f, g)-\phi\left(f^{\prime}, g^{\prime}\right)$. The kernel of $\widetilde{\phi}$ is $\widetilde{\Delta}$, hence $Z_{m-1}(X)_{\mathbb{R}}^{\text {lin }}$ is isomorphic to $\widetilde{I} / \widetilde{\Delta}$; therefore $Z_{m-1}(X)_{\mathbb{R}}^{\text {lin }} \cong K\left(\mathbb{Z}_{2}, 1\right)$.

Since $\frac{Z_{m-1}(X)_{\mathbb{R}}^{0}}{Z_{m-1}(X)_{\mathbb{R}}^{l i n}}=P i c^{0}(X)_{\mathbb{R}}$, and the group $\operatorname{Pic}^{0}(X)_{\mathbb{R}}$ is a closed subgroup of $P i c^{0}(X)$, thus a real torus, from the homotopy sequence induced by the short exact sequence

$$
0 \longrightarrow Z_{m-1}(X)_{\mathbb{R}}^{\text {lin }} \longrightarrow Z_{m-1}(X)_{\mathbb{R}}^{0} \longrightarrow P i c^{0}(X)_{\mathbb{R}} \longrightarrow 0
$$

we have

$$
\pi_{k} Z_{m-1}(X)_{\mathbb{R}}^{0}= \begin{cases}\mathbb{Z}_{2} \oplus \pi_{1} \operatorname{Pic}^{0}(X)_{\mathbb{R}}, & \text { if } k=1 \\ 0, & \text { otherwise }\end{cases}
$$

This completes the proof.

Proposition 6.3. For a nonsingular real projective variety $X, N S(X)_{\mathbb{R}}$ is finitely generated. 
Proof. Let $m$ be the dimension of $X$ and let

$$
H=\frac{Z_{m-1}(X)_{\mathbb{R}} \cap Z_{m-1}(X)^{\text {alg }}}{Z_{m-1}(X)_{\mathbb{R}}^{\text {lin }}} .
$$

By Proposition 5.17, $H$ is embedded as a closed subgroup of $P i c^{0}(X)=\frac{Z_{m-1}(X)^{a l g}}{Z_{m-1}(X)^{l i n}}$; hence $H$ is a Lie subgroup of $P i c^{0}(X)$ and therefore $\pi_{0}(H)$ is finitely generated. The inclusion map $i: \operatorname{Pic}(X)_{\mathbb{R}} \rightarrow \operatorname{Pic}(X)$ induces a map

$$
i_{*}: N S(X)_{\mathbb{R}}=\pi_{0}\left(\operatorname{Pic}(X)_{\mathbb{R}}\right) \rightarrow \pi_{0}(\operatorname{Pic}(X))
$$

whose kernel is $\pi_{0}(H)$, and because $\pi_{0}(\operatorname{Pic}(X))$ is finitely generated, $N S(X)_{\mathbb{R}}$ is finitely generated.

Let

$$
T=\left\{a-b \mid a+\bar{a}=b+\bar{b}, a, b \in \mathscr{C}_{m-1}(X)\right\} .
$$

Lemma 6.4. $T=\left\{a-\bar{a} \mid a \in \mathscr{C}_{m-1}(X)\right\}$.

Proof. Suppose that $a, b \in \mathscr{C}_{m-1}(X)$ and $a+\bar{a}=b+\bar{b}$. Write $a=\sum_{i=1}^{n} n_{i} V_{i}$ where each $V_{i}$ is an irreducible subvariety and $n_{i}>0$. Since $a-b \in T$, we may assume that $a$ and $b$ have no common irreducible subvariety components. From the relation $a+\bar{a}=b+\bar{b}$, we see that each $V_{i}$ must be a component of $\bar{b}$. Thus $b=\bar{a}$.

The following observation is the main tool that we are going to use to compute $R_{m-1}(X)$.

Proposition 6.5. We have the following exact sequences of topological groups:

(1) $0 \longrightarrow Z_{m-1}(X)_{\mathbb{R}} \hookrightarrow Z_{m-1}(X) \stackrel{S a}{\longrightarrow} T \longrightarrow 0$,

(2) $0 \longrightarrow T \hookrightarrow Z_{m-1}(X) \stackrel{A v}{\longrightarrow} Z_{m-1}(X)^{a v} \longrightarrow 0$,

where $S a(c)=c-\bar{c}$ and $A v(c)=c+\bar{c}$, and the groups $T$ and $Z_{m-1}(X)^{\text {av }}$ are isomorphic as a topological group to $\frac{Z_{m-1}(X)}{Z_{m-1}(X)_{\mathbb{R}}}$ and $\frac{Z_{m-1}(X)}{T}$ respectively.

Proof. A direct verification shows that the sequences are exact. To show that $T$ is isomorphic as a topological group to $\frac{Z_{m-1}(X)}{Z_{m-1}(X)_{\mathbb{R}}}$, it suffices to prove that $S a$ is a closed map. Let $K_{1} \subset K_{2} \subset \cdots \subset Z_{m-1}(X)$ be the canonical filtration. The topology of $T$ is the subspace topology of $Z_{m-1}(X)$. For $C \subset Z_{m-1}(X)$ a closed subset, $C \cap K_{n}$ is compact and $S a\left(C \cap K_{n}\right)=S a(C) \cap K_{2 n}$ which is closed for any $n$, so $S a$ is a closed map. The map $A v$ is a closed map which is proved in Proposition 4.2 .

Lemma 6.6. Suppose that $X$ is a nonsingular real projective variety of dimension $m$.

(1) For $k \geq 2, \pi_{k} Z_{m-1}(X)^{\text {av }}$ is a 2-torsion group.

(2) The homotopy group

$$
\pi_{k} T= \begin{cases}0, & \text { if } k>2 \\ \mathbb{Z}, & \text { if } k=2 \\ \pi_{1}\left(\frac{T}{Z_{m-1}(X)^{l i n}}\right), & \text { if } k=1\end{cases}
$$

and hence $\pi_{k} T$ is free for $k>0$.

(3) If $H^{1}(X, \mathbb{C})=0$ and $N S(X)$ is free, then $\pi_{0} T$ is free. 
Proof. (1) For a continuous map $f: S^{k} \longrightarrow Z_{m-1}(X)^{a v}$ where $k \geq 2,2 f(t) \in$ $2 Z_{m-1}(X)_{\mathbb{R}} \subset Z_{m-1}(X)^{a v}$. But since $\pi_{k}\left(2 Z_{m-1}(X)_{\mathbb{R}}\right) \cong \pi_{k}\left(Z_{m-1}(X)_{\mathbb{R}}\right)=$ 0 for $k \geq 2$, we see that $2 f$ is null homotopic. Thus $\pi_{k} Z_{m-1}(X)^{a v}$ is a 2 torsion group for $k \geq 2$.

(2) Let $T^{0}$ be the zero-component of $T$. Since $T$ is a closed subgroup of $Z_{m-1}(X), \frac{T^{0}}{Z_{m-1}(X)^{l i n}} \hookrightarrow \frac{Z_{m-1}(X)^{a l g}}{Z_{m-1}(X)^{l i n}}=P i c^{0}(X)$ is a closed embedding and hence $\frac{T^{0}}{Z_{m-1}(X)^{l i n}}$ is a closed Lie subgroup of $\operatorname{Pic}^{0}(X)$ which implies that $\pi_{k} \frac{T}{Z_{m-1}(X)^{l i n}}$ is free for $k>0$. By a similar calculation of the homotopy type of $Z_{m-1}(X)_{\mathbb{R}}^{l i n}$ in Proposition 6.2 , we get $Z_{m-1}(X)^{l i n} \cong K(\mathbb{Z}, 2)$. From the homotopy sequence induced by the short exact sequence

$$
0 \longrightarrow Z_{m-1}(X)^{l i n} \longrightarrow T \longrightarrow \frac{T}{Z_{m-1}(X)^{l i n}} \longrightarrow 0
$$

we get the result.

(3) Since $H^{1}(X, \mathbb{C})=0, \operatorname{Pic}^{0}(X)$ is trivial, hence $Z_{m-1}(X)^{\text {alg }}=Z_{m-1}(X)^{\text {lin }}$. Then $\pi_{0} Z_{m-1}(X)=\frac{Z_{m-1}(X)}{Z_{m-1}(X)^{l i n}}$ is free from the hypothesis. From the homotopy sequence induced by the short exact sequence above, we see that $\pi_{0} T$ is also free.

Theorem 6.7. For a nonsingular real projective $X$ of dimension $m, \pi_{k} R_{m-1}(X)=$ 0 for $k>2$.

Proof. Consider the two exact sequences in Proposition 6.5:

(1) $0 \longrightarrow Z_{m-1}(X)_{\mathbb{R}} \hookrightarrow Z_{m-1}(X) \stackrel{S a}{\longrightarrow} T \longrightarrow 0$,

(2) $0 \longrightarrow T \hookrightarrow Z_{m-1}(X) \stackrel{A v}{\longrightarrow} Z_{m-1}(X)^{a v} \longrightarrow 0$.

From the homotopy sequence induced by the first exact sequence, we see that $\pi_{k} T=0$ if $k>2$. And from the homotopy sequence induced by the second exact sequence, we get $0 \rightarrow \pi_{3} Z_{m-1}(X)^{a v} \stackrel{\phi_{1}}{\rightarrow} \pi_{2} T \stackrel{i_{2 *}}{\longrightarrow} \pi_{2} Z_{m-1}(X) \rightarrow \pi_{2} Z_{m-1}(X)^{a v} \stackrel{\phi_{2}}{\longrightarrow}$ $\pi_{1} T \rightarrow \pi_{1} Z_{m-1}(X) \rightarrow \cdots$. By Lemma 6.6 , the groups $\pi_{1} T, \pi_{2} T$ are free and the groups $\pi_{2} Z_{m-1}(X)^{a v}, \pi_{3} Z_{m-1}(X)^{a v}$ are 2-torsion groups, thus $\phi_{1}, \phi_{2}$ are 0 -maps and $\pi_{3} Z_{m-1}(X)^{a v}=0$.

Consider the composition of the maps: $T \stackrel{i_{2}}{\rightarrow} Z_{m-1}(X) \stackrel{\rho_{1}}{\rightarrow} T$ which is $2 i d: T \longrightarrow$ $T$, and consider the induced maps on homotopy groups: $\pi_{2} T \stackrel{i_{2 *}}{\longrightarrow} \pi_{2} Z_{m-1}(X) \stackrel{\rho_{1 *}}{\longrightarrow}$ $\pi_{2} T$. All of these groups are isomorphic to $\mathbb{Z}$ and $\rho_{1 *} \circ i_{2 *}=2 i d_{*}$ which is of degree 2. If $\rho_{1 *}: \pi_{2} Z_{m-1}(X) \longrightarrow \pi_{2} T$ is of degree 1 , from the homotopy sequence induced by the first exact sequence, we have $0 \rightarrow \pi_{2} Z_{m-1}(X) \stackrel{\rho_{1 *}}{\rightarrow} \pi_{2} T \rightarrow \pi_{1} Z_{m-1}(X)_{\mathbb{R}} \rightarrow$ $\pi_{1} Z_{m-1}(X) \rightarrow \cdots$ which implies that $\pi_{1} Z_{m-1}(X)_{\mathbb{R}}$ is mapped injectively into $\pi_{1} Z_{m-1}(X)$, which is impossible. Thus $\rho_{1 *}$ is of degree 2 and $i_{2 *}$ is of degree 1 . Since $\phi_{2}$ is a 0 -map, we have $\pi_{2} Z_{m-1}(X)^{a v}=0$.

The result now follows from the homotopy sequence induced by

$$
0 \longrightarrow Z_{m-1}(X)^{a v} \longrightarrow Z_{m-1}(X)_{\mathbb{R}} \longrightarrow R_{m-1}(X) \longrightarrow 0 .
$$

Definition 6.8. Suppose that $X$ is a nonsingular real projective variety. The Picard number $\rho(X)$ is the rank of the free part of $\pi_{0} Z_{m-1}(X)$, and we call $R N S(X)=\pi_{0} R_{m-1}(X)$ the reduced real Neron-Severi group of $X$. 
Corollary 6.9. Suppose that $X$ is a nonsingular real projective variety of dimension $m$; we have the following relation:

$$
\rho(X)+1 \equiv \operatorname{dim}_{\mathbb{Z}_{2}} R N S(X)+\operatorname{dim}_{\mathbb{Z}_{2}} \pi_{1} R_{m-1}(X)+\operatorname{dim}_{\mathbb{Z}_{2}} \pi_{2} R_{m-1}(X) \bmod 2 .
$$

Proof. By the fundamental theorem on finitely generated abelian groups,

$$
\pi_{0} Z_{m-1}(X)=\mathbb{Z}^{k} \oplus \mathbb{Z}_{2}^{t} \oplus G
$$

where $k=\rho(X), t$ is a nonnegative integer, and $G$ is in the torsion part of $\pi_{0} Z_{m-1}(X)$ where $\mathbb{Z}_{2}$ is not a direct summand of $G$.

From the homotopy sequence induced by

$$
0 \longrightarrow 2 Z_{m-1}(X) \longrightarrow Z_{m-1}(X) \longrightarrow \frac{Z_{m-1}(X)}{2 Z_{m-1}(X)} \longrightarrow 0
$$

and the homotopy groups of $Z_{m-1}(X)$, we have an exact sequence: $0 \rightarrow \pi_{2} 2 Z_{m-1}(X)$ $\rightarrow \pi_{2} Z_{m-1}(X) \rightarrow \pi_{2}\left(\frac{Z_{m-1}(X)}{2 Z_{m-1}(X)}\right) \rightarrow \pi_{1} 2 Z_{m-1}(X) \rightarrow \pi_{1} Z_{m-1}(X) \rightarrow \pi_{1}\left(\frac{Z_{m-1}(X)}{2 Z_{m-1}(X)}\right) \rightarrow$ $\pi_{0} 2 Z_{m-1}(X) \rightarrow \pi_{0} Z_{m-1}(X) \rightarrow \pi_{0}\left(\frac{Z_{m-1}(X)}{2 Z_{m-1}(X)}\right) \rightarrow 0$. The map $i_{*}: \pi_{*} 2 Z_{m-1}(X) \rightarrow$ $\pi_{*} Z_{m-1}(X)$ is easily seen to be a map of degree 2 , hence $\pi_{2}\left(\frac{Z_{m-1}(X)}{2 Z_{m-1}(X)}\right)=\mathbb{Z}_{2}$, $\operatorname{dim}_{\mathbb{Z}_{2}} \pi_{1}\left(\frac{Z_{m-1}(X)}{2 Z_{m-1}(X)}\right)=r k P i c^{0}(X)+t$, and $\operatorname{dim}_{\mathbb{Z}_{2}} \pi_{0}\left(\frac{Z_{m-1}(X)}{2 Z_{m-1}(X)}\right)=k+t$. Therefore $\sum_{n=0}^{2} \operatorname{dim}_{\mathbb{Z}_{2}} \pi_{n}\left(\frac{Z_{m-1}(X)}{2 Z_{m-1}(X)}\right)=1+r k P i c^{0}(X)+2 t+k$, and we note that the rank of the Picard variety $\operatorname{Pic}^{0}(X)$ is even. The result now follows from Theorem 6.7 and Theorem 4.6.

Corollary 6.10. Suppose that $X$ is a nonsingular projective variety of dimension $m$. We assume that $H^{1}(X, \mathbb{C})=0$ and $N S(X)$ is free. Then we have

$$
\rho(X)+1 \equiv \operatorname{dim}_{\mathbb{Z}_{2}} R N S(X)+\operatorname{dim}_{\mathbb{Z}_{2}} \pi_{1} R_{m-1}(X) \bmod 2 .
$$

Proof. By Lemma 6.6, $\pi_{0} T$ is free. Since $\operatorname{Pic} c^{0}(X)$ is trivial, $\pi_{1} Z_{m-1}(X)=0$. Consider the homotopy sequence induced by the second exact sequence of Proposition 6.5. We have

$$
\cdots \rightarrow \pi_{1} Z_{m-1}(X) \rightarrow \pi_{1} Z_{m-1}(X)^{a v} \rightarrow \pi_{0} T \rightarrow 0
$$

which implies that $\pi_{1} Z_{m-1}(X)^{a v}$ is free. From the homotopy sequence induced by the exact sequence $0 \rightarrow Z_{m-1}(X)^{a v} \rightarrow Z_{m-1}(X)_{\mathbb{R}} \rightarrow R_{m-1}(X) \rightarrow 0$ and Theorem 6.7 , we have an exact sequence $0 \rightarrow \pi_{2} R_{m-1}(X) \rightarrow \pi_{1} Z_{m-1}(X)^{a v}$, but since $\pi_{2} R_{m-1}(X)$ is 2 -torsion, this implies $\pi_{2} R_{m-1}(X)=0$.

Corollary 6.11. If $X$ is a real complete intersection of dimension $>2$, then

$$
\operatorname{dim}_{\mathbb{Z}_{2}} R N S(X) \equiv \operatorname{dim}_{\mathbb{Z}_{2}} \pi_{1} R_{m-1}(X) \bmod 2 .
$$

Proof. By the weak Lefschetz theorem and the exponential sequence on $X$, we have $N S(X) \cong \mathbb{Z}$ and $H^{1}(X, \mathbb{C})=0$, so $\rho(X)=1$. The result then follows from Corollary 6.10 .

\section{ACKNOWLEDGEMENT}

The author thanks Blaine Lawson for his encouragement and patience in listening to the proof and Christian Haesemeyer for some helpful remarks. He is also indebted to the referee for careful proofreading, corrections and suggestions, the National Center for Theoretical Sciences of Taiwan at Hsinchu for providing a wonderful working environment, and Yusuf Mustopa for help with some linguistic matters. 


\section{REFERENCES}

1. D. J. Benson, Representations and cohomology II, Cambridge studies in adv. math., 31, Cambridge, 1991. MR1156302 (93g:20099)

2. A. Degtyarev and V. Kharlamov, Topological properties of real algebraic varieties: Rokhlin's way, Russian Math. Surveys 55 (2000), no. 4, 735-814. MR1786731 (2001h:14077)

3. E. Friedlander, Algebraic cycles, Chow varieties, and Lawson homology, Compositio Math. 77 (1991), 55-93. MR1091892 (92a:14005)

4. E. Friedlander, Filtration on algebraic cycles and homology, Annales Scientifiques de l'Ecole Normale Superieure Ser. 4, 28 no. 3 (1995), p. 317-343. MR1326671 (96i:14004)

5. Y. Félix, S. Halperin, J. Thomas, Rational homotopy theory, Springer, 2000.

6. E. Friedlander and H.B. Lawson, A theory of algebraic cocycles, Annals of Math. 136 (1992), 361-428. MR1185123 (93g:14013)

7. E. Friedlander and H.B. Lawson, Moving algebraic cycles of bounded degree, Invent. Math. 132 (1998), 91-119. MR1618633 (99k:14011)

8. E. Friedlander and H.B. Lawson, Duality relating spaces of algebraic cocycles and cycles, Topology 36 (1997), 533-565. MR1415605 (97k:14007)

9. D. A. Gudkov, The toplogy of real projective algebraic varieties, Russian Math. Surveys 29, no. 4, (1974), 3-79. MR0399085 (53:2936)

10. H.B. Lawson, Algebraic cycles and homotopy theory, Annals of Math. 129 (1989), 253-291. MR986794 (90h:14008)

11. H.B. Lawson, Spaces of algebraic cycles, Surveys. Diff. Geo., 1995, Vol 2, 137-213. MR1375256 (97m:14006)

12. P. Lima-Filho, Lawson homology for quasi- projective varieties, Compositio Math. 84 (1992), 1-23. MR1183559 (93j:14007)

13. P. Lima-Filho, The topological group structure of algebriac cycles, Duke Math. J., 75 (1994), no. 2, 467-491. MR1290199 (95i:14010)

14. P. Lima-Filho, Completions and fibrations for topological monoids, Trans. AMS, 340 (1993), no. 1, 127-147. MR1134758 (94a:55009)

15. H.B. Lawson, P. Lima-Filho, M. Michelsohn, Algebraic cycles and the classical groups I: real cyclces, Topology, 42, (2003) 467-506. MR1941445 (2003m:14013)

16. P. Samuel, Méthodes d'algèbre abstraite en géométrie algébrique, Ergebnisse der math., Springer-Verlag, 1955. MR0072531 (17:300b)

17. Norman E. Steenrod, A convenient category of topological spaces, Michigan Math. J. 14 (1967), 133-152. MR0210075 (35:970)

18. Jyh-Haur Teh, A homology and cohomology theory for real projective varieties, preprint in Arxiv.org, math.AG/0508238.

19. R. Thom, "Sur l'homologie des varietés algèbriques réelles", Diff. and comb. topology, Princeton Univ. Press, Princeton-New York 1965, pp. 255-265. MR0200942 (34:828)

20. Andre Weil, On Picard varieties, Amer. J. Math., Vol. 74, No. 4, 865-894. MR0050330 $(14: 314 \mathrm{e})$

Department of Mathematics, National Tsing Hua University of Taiwan, No. 101, Kuang Fu Road, Hsinchu, 30043, Taiwan

E-mail address: jyhhaur@math.nthu.edu.tw 